\title{
From relativistic time dilation to psychological time perception: an approach and model, driven by the theory of relativity, to combine the physical time with the time perceived while experiencing different situations.
}

\author{
Andrea Conte $\mathrm{e}^{1, *}$
}

\begin{abstract}
An approach, supported by a physical model driven by the theory of relativity, is presented. This approach and model tend to conciliate the relativistic view on time dilation with the current models and conclusions on time perception. The model uses energy ratios instead of geometrical transformations to express time dilation. Brain mechanisms like the arousal mechanism and the attention mechanism are interpreted and combined using the model. Matrices of order two are generated to contain the time dilation between two observers, from the point of view of a third observer. The matrices are used to transform an observer time to another observer time. Correlations with the official time dilation equations are given in the appendix.
\end{abstract}

Keywords: Time dilation, Time perception, Definition of time, Lorentz factor, Relativity, Physical time, Psychological time, Psychology of time, Internal clock, Arousal, Attention, Subjective time, Internal flux, External flux, Energy system

\footnotetext{
*Corresponding author

Email address: conte.an@hotmail.com (Andrea Conte)

${ }^{1}$ Declarations of interest: none
} 


\section{Contents}

1 Introduction 3

1.1 The unit of time . . . . . . . . . . . . . . . . . 4

1.2 The Lorentz factor . . . . . . . . . . . . . . . . . . 6

2 Physical model $\quad 7$

2.1 Energy system . . . . . . . . . . . . . . . . . . . 7

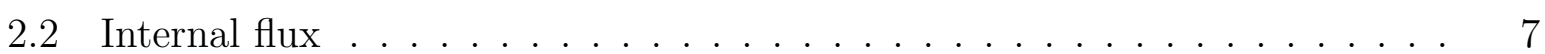

2.3 Internal flux ratio . . . . . . . . . . . . . . . . . . . . . . . . 9

2.4 Non-isolated system interaction . . . . . . . . . . . . . . . . . . 10

2.5 External flux . . . . . . . . . . . . . . . . . . . . . . . . 11

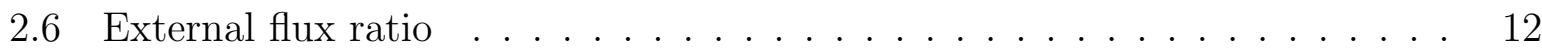

2.7 Total flux . . . . . . . . . . . . . . . . . . . . . . 12

2.8 Derivation to obtain the Lorentz factor denominator . . . . . . . . . . . . . . 14

2.9 Clock flux . . . . . . . . . . . . . . . . . . . 15

2.10 Transformation matrices . . . . . . . . . . . . . . . . 15

3 Internal and external fluxes of brain mechanisms $\quad 16$

3.1 The internal clock . . . . . . . . . . . . . . . . . . . . 17

3.2 Arousal mechanism . . . . . . . . . . . . . . . . . . . . . . . 18

3.3 Attention mechanism . . . . . . . . . . . . . . . . . . . . 19

3.4 Specific attention mechanism . . . . . . . . . . . . . . . 20

3.5 Combination of arousal, attention and specific attention mechanisms . . . . 22

4 Combining physical time dilation and psychological time perception 23

4.1 Parameters . . . . . . . . . . . . . . . . . . . . . . 23

4.2 Step 1 - Finding the internal and external fluxes . . . . . . . . . . . . 25

4.3 Step 2 - Generating the transformation matrices . . . . . . . . . . . . 28

4.4 Step 3 - Applying the transformations . . . . . . . . . . . . . . . . . 32

4.5 Results . . . . . . . . . . . . . . . . . . . . . 34

5 Conclusion $\quad 38$

$\begin{array}{lll}\text { Appendix A Calculations step } 1 \text { and flux ratio tables } & 39\end{array}$

Appendix A.1 Intermediate calculations step 1 . . . . . . . . . . . . . 39

Appendix A.2 Flux ratio tables . . . . . . . . . . . . . . . . . 41

Appendix B Correlation with the time dilation formulas 44

Appendix B.1 Lorentz factor correlation . . . . . . . . . . . . . . . 44

Appendix B.2 Gravitational time dilation correlation . . . . . . . . . 46

$\begin{array}{llll}\text { Appendix C } & \text { Supplementary data } & 48\end{array}$ 


\section{Introduction}

Different models and methods have been proposed to express how the perceived time is influenced by brain mechanisms (Treisman, 1963; Gibbon et al., 1984; Hass and Durstewitz, 2016; Mioni et al., 2020). From these models, the notion of subjective time emerged (Thönes and Stocker, 2019), which goes in pair with the assumption of the existence of an internal clock (Treisman, 1963; Church, 1984; Fayolle et al., 2015). Some models relate the psychology of time to physics theories (Buhusi and Meck, 2009; Kent et al., 2019; Ghaderi, 2019). In these latter models, ratios are used to interpret the acceleration or deceleration of time such as the ratio between hopelessness and the arousal (Kent et al., 2019) or the ratio between the entropy inside the brain and the entropy outside the brain (Ghaderi, 2019). These ratios behave similarly to the Lorentz factor used to calculate time dilation in Special Relativity (Einstein, 1905b). Aside from the existing models, experiences have been made to compare the passage of time felt during different situations or tasks (DroitVolet, 2013; Davydenko and Peetz, 2017; Edwards and McCormick, 2017; Arstila, 2012). All these different views on time perception consolidate the motivation in presenting the approach and the physical model described in this paper. This approach use the principles and concepts found in Einstein's theories of Special and General Relativity concerning time dilation (Einstein, 1920), to apply them on time perception. The model is used in section 4 , assisted by transformation matrices, to combine the physical time dilation with the psychological time perception. The intent of this approach is to offer a supplementary physical perspective on time perception. It is not aimed at replacing any of the current approved models on time perception. Before presenting the model, the definition of the unit of time is introduced and examined in the next section, followed by a section interpreting the Lorentz factor. 


\subsection{The unit of time}

Human beings developed the notion of time and created the concept of clock to assist in estimating the duration spent in daily activities. Without it, it would not be possible to correctly estimate the passage of time (Davydenko and Peetz, 2017; Xu and David, 2018). This concept is well established in science since its existence. All classical physics equations were based on it and on the postulate that time was absolute and flowing at the same regular intervals in all reference frames (Capek, 1987; Thomas, 2015). However, A. Einstein demonstrated the contrary in his theory of Special Relativity (Einstein, 1905b). He showed that time can shrink or dilate between reference frames. To demonstrate his thoughts, he used the geometry of light travelling through space as a tool (Einstein, 1920). At this epoch, the definition of time and its notion was not the same as today. When his paper was published, the unit of second was defined from the earth rotational movements (Taylor et al., 2008). Nowadays, time is still related to movements but its definition is different. The unit of second is defined as the number of oscillations of a natural oscillator when light travels a chosen distance. The oscillator makes 9192631770 cycles as soon as the light has reached the distance of 299792458 meters (Taylor et al., 2008).

$$
9192631770 \text { cycles } \Leftrightarrow 1 \text { second }
$$

The ratio between the cycles and the distance gives the number of cycles made when light has reached one meter.

$$
\frac{9192631770 \text { cycles }}{299792458 \mathrm{~m}}=30.6633189885 \text { cycles } / \mathrm{m}
$$

This means when 30.6633189885 cycles is made, light has reached 1 meter.

$$
30.6633189885 \text { cycles } \Leftrightarrow 1 \text { meter }
$$

The unit of meter is officially defined as being $1 / 299792458$ of the distance that light travels in 1 second (Taylor et al., 2008).

The official definition of time and distance, based on the speed of light, uses the concept of cycle to define time. From a mechanical perspective, a cycle is a motion. To generate this motion, a work is needed. In mechanics, a work is the energy needed to create a displacement over a certain distance. The unit of work is the Joule which is also the unit of energy (Taylor et al., 2008). In a logical sequence, work implies motion that implies cycle that implies the definition of a second.

$$
\text { Work } \Rightarrow \text { Motion } \Rightarrow \text { Cycle } \Rightarrow \text { Second }
$$


These implications show that time and time perception by consequence, are dependent on motion which depends on the energy that brought this motion to live. A. Einstein already expressed this dependency in his book "Relativity: The Special and General Theory". In the following excerpt, he used the expression "time is robbed of its independence" to emphasize the fact that he saw time not to be an independent variable but a variable dependent on motion.

"That we have not been accustomed to regard the world in this sense as a four-dimensional continuum is due to the fact that in physics, before the advent of the theory of relativity, time played a different and more independent rôle, as compared with the space co-ordinates. It is for this reason that we have been in the habit of treating time as an independent continuum. As a matter of fact, according to classical mechanics, time is absolute, i.e. it is independent of the position and the condition of motion of the system of co-ordinates. We see this expressed in the last equation of the Galileian transformation $\left(t^{\prime}=t\right)$. The four-dimensional mode of consideration of the "world" is natural on the theory of relativity, since according to this theory time is robbed of its independence. This is shown by the fourth equation of the Lorentz transformation: $t^{\prime}=\frac{t-\frac{v}{c^{2}} x}{\sqrt{1-\frac{v^{2}}{c^{2}}}} "($ Einstein, 1920)

Following the implications of equation (4), if the energy varies (i.e. become stronger or weaker), the intensity of the work will also vary, making the motion faster or slower, causing a variation in the unit of time definition. It is similar to electric variations appearing on a light bulb. When the tension varies, the light shines brighter or dimmer. The same principle is applied in the theory of General Relativity (Einstein, 1916). Someone near a strong energy point, like a black hole, will have their time slowed down. In this regard, time seems plastic and influenced by energy fluctuations. General relativity has been the official candidate theory to demonstrate these principles.

By manipulating the level of energy used to provide a work, it is possible to adjust what is dependent on this work, like the energy provided to make ticking a clock. A clock is dependent on the work provided by batteries or other mechanisms. Our perception of time is also dependent on mechanisms such as the arousal and the attention mechanisms which are themselves dependent on deeper brain mechanisms like the orchestration of neurons which is itself dependent on motion of atoms influenced by external energy. In the objective world, we use clocks to synchronize our events or activities. Inside the brain, in the subjective world, it has been observed that functions synchronize their activities following one or maybe more internal clocks (Buhusi and Meck, 2009). Section 3 gives a relativistic view on the internal clock as well as a relativistic view on the arousal and attention mechanisms. 


\subsection{The Lorentz factor}

The Lorentz factor is the official formula that is used to demonstrate and calculate time dilation for objects or atomic particles in motion. The Lorentz factor is derived by using the Lorentz transformations (Einstein, 1920) which are geometrical transformations. In his paper on Special Relativity, A. Einstein used another strategy to arrive at the Lorentz factor, giving it a stronger significance. Time dilation is, since then, represented by the Lorentz factor.

$$
\begin{aligned}
& \text { Lorentz factor }=\frac{1}{\sqrt{1-\frac{v^{2}}{c^{2}}}} \\
& \text { Lorentz factor denominator }=\sqrt{1-\frac{v^{2}}{c^{2}}}
\end{aligned}
$$

The letter $v$, in both equations (5) and (6), represents the relative speed between two frames of reference. The letter $c$ is the speed of light, the maximum reachable speed. In the denominator part represented by equation (6), the more the speed $v$ approaches the speed of light $c$, the more the result value will tend to zero. The division made in equation (5) inverses the signification. The more the speed $v$ approaches the speed of light $c$, the more the result will tend to positive infinity. Both significations are correct, it will depend on the context of usage.

In a first context, a person $\mathrm{A}$ is at rest and a person $\mathrm{B}$ is in motion at the speed $v$. Another observer $\mathrm{C}$ is at rest looking at the scene. Using equation (5), the observer $\mathrm{C}$ sets $v$ to be the speed of person B. The result will be the time of person A's clock that has elapsed when 1 second has elapsed on person B's clock. The elapsed time on person A's clock will be higher than on person B's clock. This means person B's clock will tick slower. Observer C will observe a time dilation of person B's clock.

In a second context, which is the same situation of the first context, a person $\mathrm{A}$ is at rest and a person $\mathrm{B}$ is in motion at the speed $v$ while an observer $\mathrm{C}$ is at rest looking at the scene. Using only the denominator part represented by equation (6), the result calculated by Observer $\mathrm{C}$ will give the duration that has elapsed on person B's clock when 1 second has elapsed on person A's clock. This duration will be lower than person A's clock. Person B's clock will tick slower than person A's clock. Observer $\mathrm{C}$ will observe a time dilation on person B's clock.

In both contexts, when the speed of person B is faster, person B's clock will tick slower than person A's clock. 


\section{Physical model}

The model presented in this section is a theoretical model based on energy ratios. These ratios produce results which correlate with the time dilation principles found in Special and General Relativity as demonstrated in Appendix B. The model is used in section 3 to give a relativistic view of the arousal and attention mechanisms involved in time perception. The model is based on the concept of energy system, the concepts of internal and external fluxes of energy systems and the ratios between these fluxes. All these concepts are defined in this section.

\subsection{Energy system}

An energy system is the chosen term in this paper to represent any object or concept of any size that has a potential energy like an atomic particle, a neuron, a brain, a person, a spaceship or even the earth when the focus is put on their internal and external energetic behaviors and transfers. An energy system can be isolated from interactions or can interact with its environment where transfers of energy are made. In this latter case the system is said to be non-isolated. An energy system has an internal flux and an external flux defined in the next sections.

\subsection{Internal flux}

An energy system has an internal flux. To define the internal flux, Ohm's law is used as a starting point. From Ohm's law, the intensity in an electric current is proportional to the tension producing that current and is inversely proportional to the resistance in which the current flows.

$$
\text { Intensity }=\frac{\text { Tension }}{\text { Resistance }}
$$

This equation has a more general meaning than just being useful in electricity. In nature, similar behaviors are met. Water flowing in a river has a stream intensity while the ground, gravity and other factors apply a resistance to the stream. Another example is an electric car accelerating against a strong wind. The car momentum can be viewed as the intensity while the wind and other factors apply a resistance. The car battery has a potential energy or tension. The stronger the wind (Resistance), the more an acceleration is required to request more energy (Tension) to keep the same momentum (Intensity). The gravity effect can also be regarded as including the concepts of intensity, tension and resistance. A. Einstein introduced his Theory of General Relativity in his book (Einstein, 1920) by using a construct similar to equation (7). 
"According to Newton's law of motion, we have

$($ force $)=($ inertial mass $) *($ Acceleration $)$,

where the "inertial mass" is a characteristic constant of the accelerated body.

If now gravitation is the cause of the acceleration, we then have

$($ force $)=($ gravitational mass $) *($ intensity of the gravitational field $)$,

where the "gravitational mass" is likewise a characteristic constant for the body"

(Einstein, 1920)

Using the equation he formulated, the terms can be rearranged by putting the intensity in evidence.

$$
\text { Intensity of the gravitational field }=\frac{\text { Force }}{\text { Gravitational mass }}
$$

In this equation, the Intensity of the gravitational field is obviously regarded as the Intensity. The Force is regarded as the Tension and the Gravitational mass as the Resistance. Following these general principles of intensity, resistance and tension, equation (7) can be adapted by putting the focus on energy. To achieve this, an energy dimension is added to the tension and the term Internal is added in front of each term.

$$
\text { Internal intensity }=\frac{\text { Internal tension energy }}{\text { Internal resistance }}
$$

This relation represents the internal intensity of an isolated energy system. The following relation is the same as equation (9) except that the terminology is adapted while keeping the same meaning.

$$
\text { Internal intensity }=\frac{\text { Internal energy }}{\text { Internal resistivity }}
$$

The next step is to choose the units of measurement. Joule is the unit of energy. Therefore, the unit of Internal energy will be in Joule. In mechanics, the resistivity property of an object is expressed by its mass, then the kilogram is chosen as the unit of Internal resistivity.

$$
\text { Internal energy } \rightarrow \text { Joule } \quad \text { Internal resistivity } \rightarrow k g
$$

The unit of Joule can be converted into the base units of $\mathrm{kg} \mathrm{m}^{2} / \mathrm{s}^{2}$. After converting equation (10) to the base units, the Internal intensity will be in $m^{2} / s^{2}$.

$$
\text { Internal intensity }=\frac{\text { Internal energy }}{\text { Internal resistivity }} \Leftrightarrow \frac{\text { Joule }}{\mathrm{kg}}=\frac{\mathrm{kg} \mathrm{\textrm {m } ^ { 2 }}}{\mathrm{kg} \mathrm{s^{2 }}}=\frac{\mathrm{m}^{2}}{\mathrm{~s}^{2}}
$$

Applying a square root to the Internal intensity, changes the unit in $m / s$.

$$
\sqrt{\text { Internal intensity }} \Leftrightarrow \sqrt{\frac{m^{2}}{s^{2}}}=\frac{m}{s}
$$


The interpretation of this unit of speed could be confusing and may contrast to common sense. This result must not be interpreted as a speed but interpreted as an indicator of the Internal intensity.

The unit of $\mathrm{m} / \mathrm{s}$ means doing a displacement of one meter per one second period. Because the term displacement is less appropriate in this case, the term flux will be used. A flux is the passage of a quantity of something per one second period. The term Internal flux seems an adequate candidate to name this unit. The internal flux is the square root of the internal intensity and its unit is $m / s$. To avoid the confusion, the term $f_{m} / s$ will be used instead of $\mathrm{m} / \mathrm{s}, f$ standing for $f l u x$. This is only to indicate that the interpretation must be taken as a flux indicator instead of a simple speed.

$$
\text { Internal flux }=\sqrt{\text { Internal intensity }} \Leftrightarrow \sqrt{\frac{m^{2}}{s^{2}}}=\frac{m}{s} \Leftrightarrow f_{m / s}
$$

For convenience and flexibility purpose, a conversion from the unit of Joule into the unit of $M c^{2}$ will be used implicitly from this point forward. This conversion is possible by using the Einstein's mass-energy equivalence equation.

$$
E=M c^{2}
$$

This equation defines the potential energy of a mass. A mass can be converted into energy and inversely (Einstein, 1905a). $E$ stands for energy in Joule, $M$ for mass in kilogram and $c^{2}$ is the speed of light in $\mathrm{m} / \mathrm{s}$ but squared. Using this equivalence, a conversion from Joule to $M c^{2}$ can be made into equation (12) while maintaining the final unit of $\mathrm{m}^{2} / \mathrm{s}^{2}$.

$$
\text { Internal intensity }=\frac{\text { Internal energy }}{\text { Internal resistivity }} \Leftrightarrow \frac{\text { Joule }}{M}=\frac{M c^{2}}{M}=\frac{M}{M}\left(\frac{m}{s}\right)^{2}=\frac{m^{2}}{s^{2}}
$$

\subsection{Internal flux ratio}

The internal flux ratio is a ratio between two internal fluxes of the same or different energy systems. It can be seen as comparing two energy states.

$$
\text { Internal flux ratio }=\frac{\text { Internal flux }}{1}=\frac{\sqrt{\text { Internal intensity }_{1}}}{\sqrt{\text { Internal flux } \text { Intensity }_{2}}} \Leftrightarrow \frac{\text { State }_{1}}{\text { State }_{2}}
$$


From the conversion used in equation (16), the equation (17) can be represented by the following variables and units.

$$
\frac{\text { Internal } \text { flux }_{1}}{\text { Internal flux }}=\frac{\sqrt{\text { Internal intensity } 1}}{\sqrt{\text { Internal intensity }} 2}=\frac{\sqrt{\frac{M_{1} c^{2}}{M_{1}}}}{\sqrt{\frac{M_{2} c^{2}}{M_{2}}}} \Leftrightarrow \sqrt{\frac{\frac{m^{2}}{s^{2}}}{\frac{m^{2}}{s^{2}}}}
$$

The internal flux ratio is used to detect a difference between two internal fluxes of the same or different systems. If the ratio value is 1 , then no difference is present because both systems have the same internal flux.

Appendix $\mathrm{B}$ demonstrates that physical time dilation can be found between two situations using an internal flux ratio.

\subsection{Non-isolated system interaction}

When an energy system is not isolated anymore, the system starts interacting with its environment. This interaction can be represented quantitatively using an energy value. The Acting energy is the chosen term to represent this energy value. When an interaction is made, the system is put into a non-isolated state. This state is represented using equation (10) by subtracting an Acting energy from the Internal energy.

$$
\begin{aligned}
& \text { Internal intensity }=\frac{\text { Internal energy }- \text { Acting energy }}{\text { Internal resistivity }} \\
& \text { Internal flux }=\sqrt{\frac{\text { Internal energy }- \text { Acting energy }}{\text { Internal resistivity }}}
\end{aligned}
$$

We can compare a non-isolated system state with an isolated system state using an internal flux ratio. If the Acting energy is greater than 0, the ratio will be less than 1 .

$$
\text { Ratio }=\sqrt{\frac{\text { State }_{1}}{\text { State }_{2}}}=\frac{\sqrt{\frac{\text { Internal energy }- \text { Acting energy }}{\text { Internal resistivity }}}}{\sqrt{\frac{\text { Internal energy }}{\text { Internal resistivity }}}} \Leftrightarrow \frac{\text { Non Isolated }}{\text { Isolated }}
$$

This ratio gives the physical time dilation between two states of the same or different systems as demonstrated in Appendix B.1. 


\subsection{External flux}

The external flux is an indicator that shows the strength of an interaction. For example, when an object is in motion, the indicator will show how strong or weak the interaction is. The higher the motion, the stronger the external flux. To define the external flux, an external intensity equation is first defined.

$$
\text { External intensity }=\frac{\text { External energy }}{\text { External resistivity }}
$$

The External intensity, External energy and External resistivity have the same units as their internal counterparts. The External flux is the square root of the External intensity. Its unit is also $m / s$.

$$
\text { External flux }=\sqrt{\text { External intensity }} \Leftrightarrow \sqrt{\frac{m^{2}}{s^{2}}}=\frac{m}{s} \Leftrightarrow f_{m / s}
$$

When an interaction is made, the system is brought into a non-isolated state. This state is represented by subtracting an opposed representation of the Acting energy, which is simply called the Not acting energy. The Not acting energy can be seen as an internal potential energy while the Acting energy can be seen as a external interacting energy. The External energy and its counterpart the Internal energy have the same initial value of $M c^{2}$. The following equations represent the interaction from the perspective of the external intensity and the external flux.

$$
\begin{aligned}
& \text { External intensity }=\frac{\text { External energy }- \text { Not acting energy }}{\text { External resistivity }} \\
&=\frac{\text { External energy }-(\text { Internal energy }- \text { Acting energy })}{\text { External resistivity }} \\
&=\frac{\text { External energy }- \text { Internal energy }+ \text { Acting energy }}{\text { External resistivity }} \\
&=\frac{M c^{2}-M c^{2}+\text { Acting energy }}{\text { External resistivity }} \\
&=\frac{\text { Acting energy }}{\text { External resistivity }} \\
& \text { External flux }=\sqrt{\frac{\text { Acting energy }}{\text { External resistivity }}}
\end{aligned}
$$




\subsection{External flux ratio}

Similarly to an Internal flux ratio, an External flux ratio is created by comparing two external fluxes.

$$
\begin{aligned}
& \text { External } \text { flux }_{1}=\sqrt{\text { External intensity } 1}=\sqrt{\frac{\text { Acting energy }_{1}}{\text { External resistivity } 1}} \\
& \text { External } \text { flux }_{2}=\sqrt{\text { External intensity } 1}=\sqrt{\frac{\text { Acting energy }_{2}}{\text { External resistivity }}} \\
& \text { External flux ratio }=\sqrt{\frac{\frac{\text { Acting } \text { energy }_{1}}{\text { External resistivity }}}{\frac{\text { Acting energy }}{2}}}
\end{aligned}
$$

\subsection{Total flux}

It exists a dependency between the External flux and the Internal flux. When the external flux is high, the internal flux is low. Inversely, when the external flux is low, the internal flux is high. A mathematical relation expresses this dependency in the form of a Pythagorean equation.

$$
\text { Total flux }{ }^{2}=\text { Internal flux }{ }^{2}+\text { External flux }{ }^{2}
$$

In this equation, the total flux is a constant. This equation is derived using equation (20) in which the Acting energy is replaced by $M v^{2} . M v^{2}$ is the chosen Acting energy when a system travels at the speed $v$. Section 2.8 shows why $M v^{2}$ was chosen.

$$
\begin{aligned}
\text { Internal } f l u x & =\sqrt{\frac{M c^{2}-\text { Acting energy }}{M}} \\
\text { Internal flux } & =\sqrt{\frac{M c^{2}-M v^{2}}{M}} \\
\text { Internal flux } x^{2} & =\frac{M c^{2}-M v^{2}}{M} \\
\text { Internal flux } x^{2} & =\frac{M}{M}\left(\frac{c^{2}}{1}-\frac{v^{2}}{1}\right) \\
\text { Internal flux } x^{2} & =c^{2}-v^{2} \\
c^{2} & =\text { Internal flux }{ }^{2}+v^{2} \\
c^{2} & =\text { Internal flux } x^{2}+\text { External flux }{ }^{2} \\
\text { Total flux } x^{2} & =\text { Internal flu } x^{2}+\text { External flux }{ }^{2}
\end{aligned}
$$


In this derivation, $c$ has been renamed Total flux and $v$ has been renamed External flux. Because a squared flux is an intensity, equation (27) can also be represented using intensities.

$$
\text { Total intensity }=\text { Internal intensity }+ \text { External intensity }
$$

The Total flux ${ }^{2}$ is a constant with value $c^{2}$. This equation shows the dependency between the External flux and the Internal flux. If one is modified, the other is modified to keep the value of $c^{2}$ constant. Fig. 1 . shows a trigonometric circle in which a rectangular triangle is represented. The hypotenuse which represents the Total flux stays constant while the other sides (External flux and Internal flux) vary accordingly to the point selected on the circle.

Fig. 1. Representation of Total flux ${ }^{2}=$ Internal flux ${ }^{2}+$ External flux ${ }^{2}$

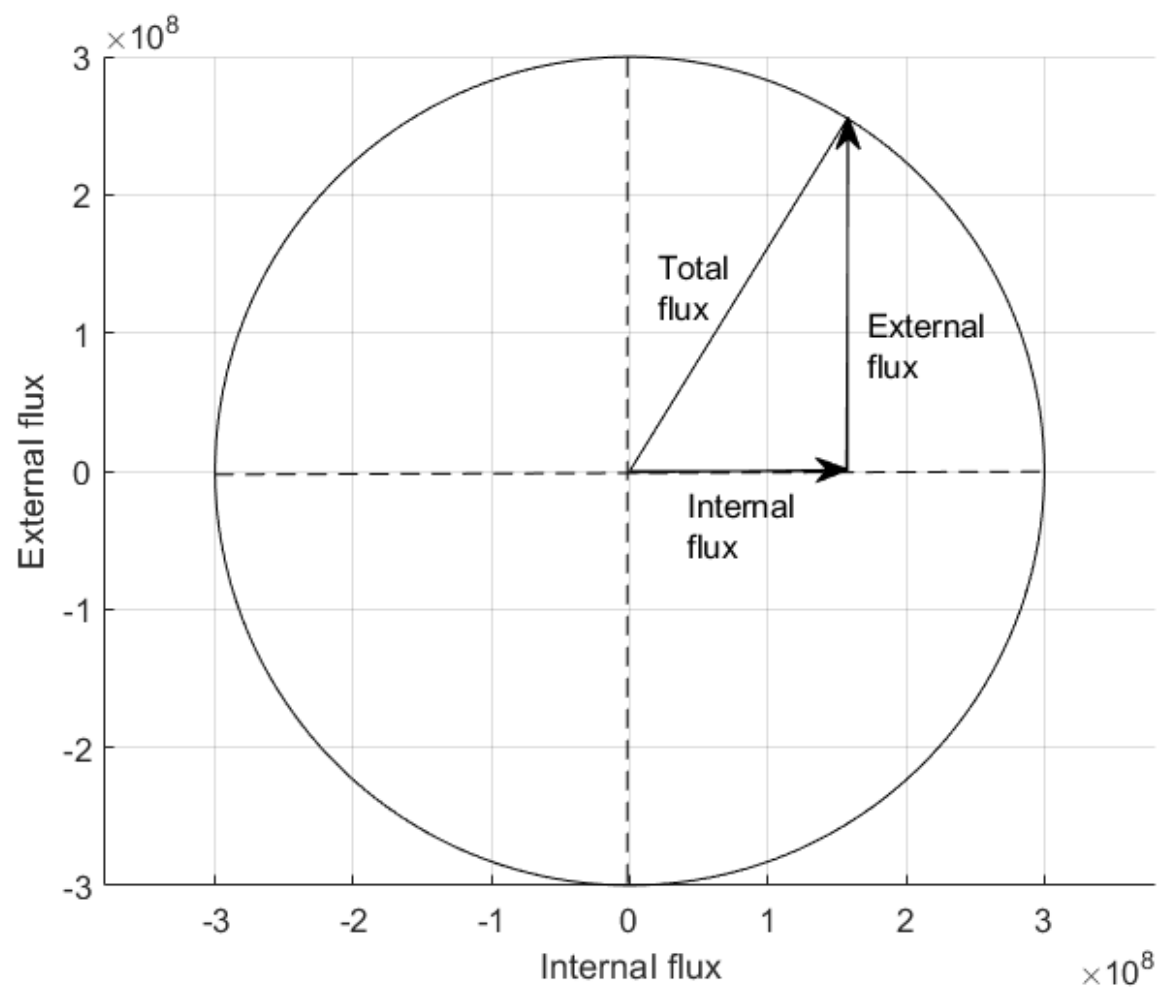




\subsection{Derivation to obtain the Lorentz factor denominator}

To obtain the Lorentz factor denominator, the equation (21) can be derived by comparing two systems, one in motion and one at rest. An Acting energy of $M v^{2}$ is set for the system in motion.

$$
\begin{aligned}
& \text { Internal flux } \text { motion }=\sqrt{\frac{M_{1} c^{2}-M_{1} v^{2}}{M_{1}}} \\
& \text { Internal flux } r e s t=\sqrt{\frac{M_{2} c^{2}}{M_{2}}} \\
& \frac{\text { Internal flux } \text { motion }_{\text {Internal flux }} \text { rest }}{\text { Int }}=\frac{\sqrt{\frac{M_{1} c^{2}-M_{1} v^{2}}{M_{1}}}}{\sqrt{\frac{M_{2} c^{2}}{M_{2}}}}=\sqrt{\frac{\frac{M_{1} c^{2}}{M_{1}}-\frac{M_{1} v^{2}}{M_{1}}}{\frac{M_{2} c^{2}}{M_{2}}}} \\
& =\sqrt{\frac{c^{2}-v^{2}}{c^{2}}}=\sqrt{\frac{c^{2}}{c^{2}}-\frac{v^{2}}{c^{2}}} \\
& =\sqrt{1-\frac{v^{2}}{c^{2}}}=\text { Lorent } z \text { factor denominator }
\end{aligned}
$$

The internal flux ratio can be seen as an extension of the Lorentz factor, this latter being implicitly included. The internal flux ratio extends the Lorentz factor by considering the mass and energy of two systems while the Lorentz factor considers only the difference in speed between two systems which is enough in the domain of Special Relativity. 


\subsection{Clock flux}

A clock can be considered as an energy system. A clock could tick faster by applying more work on it. In this case, the work energy is considered the Acting energy. The more Acting energy, the more work is done on the system. But also, if more Acting energy is present, less Internal energy is left as shown by equation (20). We use equations (20) and (25) to represent the internal and external fluxes of a clock.

$$
\begin{aligned}
& \text { Clock External flux }=\sqrt{\frac{\text { Acting energy }}{\text { Clock resistivity }}} \\
& \text { Clock Internal flux }=\sqrt{\frac{\text { Internal energy - Acting energy }}{\text { Clock resistivity }}}
\end{aligned}
$$

It is important to highlight that the External flux is how the clock will be perceived visually. The more External flux, the faster the clock will tick but the internal flux will slow down. The effect of the Internal flux is supposed to be not visible externally, this will happen inside the mechanics of the clock at the atomic level.

The internal clock model uses the term internal to pinpoint the internal part of the brain. The term internal used in internal clock must not be confused with the term internal used in internal flux. The internal clock's External flux as well as the internal clock's Internal flux have the meaning as explained using equations (31) and (32).

\subsection{Transformation matrices}

Transformation matrices can be generated using the concepts found in this model. These matrices can be used to calculate the physical time dilation and the psychological time perception between two observers. The calculations are done by a third observer, being the main frame of reference. Normally, every observer has its own frame of reference. However, comparing two observers using their own frame of reference can lead to the twin paradox (Shuler Jr., 2014). The twin paradox happens when two observers try to understand which one is in motion compared to the other. It is impossible to tell from their own point of view following only basic relativistic principles. Mathematical solutions already exist to solve this paradox. In the context of this paper, a third point of reference will be used to avoid this paradox.

The transformation matrices will not be defined in this section. They will be explained and used in section 4 . 


\section{Internal and external fluxes of brain mechanisms}

Approaches have already been made to give a relativistic dimension to brain mechanisms (Buhusi and Meck, 2009; Kent et al., 2019). The approach presented in this paper push further this view by formulating equations that could be used to approximate the time dilation between different situations. Section 4 uses these equations inside an example to approximate the time dilation experienced during different situations. This section concentrates on the representation of brain mechanisms using the concept of internal flux and external flux defined in the model section 2 .

Following the model described in section 2, the brain can be seen as an energy system. The brain consumes continuously a great quantity of energy to process its functions (Magistretti and Allaman, 2015). Equations (20) and (25) could be used to represent the Internal flux and the External flux of the brain in its entirety. The Acting energy can be replaced by the term Processing energy which would be considered all the energy consumed by the brain while processing functions. The following two equations show these representations.

$$
\begin{aligned}
& \text { Brain Internal flux }=\sqrt{\frac{\text { Internal energy - Processing energy }}{\text { Brain resistivity }}} \\
& \text { Brain External flux }=\sqrt{\frac{\text { Processing energy }}{\text { Brain resistivity }}}
\end{aligned}
$$

Representing the fluxes of the brain in its entirety might not be useful. Instead, specific mechanisms dedicated to one or many functions could be represented as shown in the following two equations.

$$
\begin{aligned}
& \text { Mechanism Internal flux }=\sqrt{\frac{\text { Internal energy }- \text { Processing energy }}{\text { Mechanism resistivity }}} \\
& \text { Mechanism External flux }=\sqrt{\frac{\text { Processing energy }}{\text { Mechanism resistivity }}}
\end{aligned}
$$

These specific mechanisms interact with each other and exchange energy in many forms. The internal fluxes and external fluxes of these mechanisms could be viewed as indicators of their interactions. 


\subsection{The internal clock}

In the brain's internal clock model, the speed of the clock is proportional to the person's arousal. Higher arousal accelerates the clock during a frightening situation (Fayolle et al., 2015). The arousal mechanism generates pulses that enter into an accumulator. Higher arousal provokes more pulses entering into the accumulator. The images of a scene will be recorded into memory proportionally to the number of pulses accumulated. When these images is read back during a recall process, the time is perceived longer because the reading speed is the same whatever number of images are recorded. More images will make the sequence longer.

The time is also perceived lasting longer during a mindfulness meditation task (DroitVolet et al., 2015) or in a relaxing task like walking in nature (Davydenko and Peetz, 2017). In these two latter tasks, the external stimuli are diminished, making the attentional resource more available. The more attentional resource available, the more the time appears elongated (i.e. dilated). However, when the attentional resource is used to concentrate on a specific task even while meditating, the time seems to accelerate (Droit-Volet et al., 2018; $\mathrm{Xu}$ and David, 2018).

To integrate these different conclusions into the model, an analogy with a computer scheduling algorithm is used. In a basic operating system scheduler, processes are treated and sent to the processor by priority and by depending on the resource available (Tanenbaum and Bos, 2014). Higher priority processes will obtain more processor resources than lower priority processes. If resources are not available to treat higher priority processes because other processes use them, then the operating system will pause these lower priority processes, will liberate resources, and will assign these new resources to the higher priority processes. Priority and resources availability are an essential part of an optimal multitasking system.

Inside the brain, resources could be considered as the available energy needed to execute processes. A high priority process could be triggered by a dangerous situation which requests a high level of energy to treat this situation. A simple task like reading a book, doing nothing or walking would suggest different levels of priority and would request different levels of energy. The available energy is considered limited to the possibility offered by the brain.

This approach and analogy will be used in the next sections to interpret the arousal and attention mechanisms. 


\subsection{Arousal mechanism}

Following the analogy of an operating system scheduler described in section 3.1, a supposition is made that the arousal mechanism triggers a high priority process to request energy when needed. This process would be triggered in order to be able to react to a frightening situation. The energy requested by the arousal mechanism will be called the Arousal energy. The requested Arousal energy might be used to send more pulses, to record more images, or to call other brain functions. The external flux equation (25) is used to represent the energy requested by the arousal mechanism.

$$
\text { Arousal external flux }=\sqrt{\frac{\text { Arousal energy }}{\text { Arousal resistivity }}}
$$

Next, by using equation (26), two states can be compared. In the first state, the requested energy is high. In the second state, the requested energy is low.

$$
\begin{aligned}
\text { Arousal external flux } 1 & =\sqrt{\frac{\text { Arousal energy high }}{\text { Arousal resistivity }}} \\
\text { Arousal external flux } 2 & =\sqrt{\frac{\text { Arousal energy low }}{\text { Arousal resistivity }}} \\
\text { Ratio } & =\frac{\text { Arousal external flux } 1}{\text { Arousal external flux } 2} \Rightarrow \text { Ratio }>1
\end{aligned}
$$

The ratio will be higher than 1 . Using the signification of the clock external flux from section 2.9, the internal clock will tick faster when the arousal is high (i.e. more images will be recorded). However, the person will perceive the time longer when the arousal increases. To represent this perception of time dilation, the signification of the clock internal flux section 2.9 is used.

$$
\text { Arousal internal flux }=\sqrt{\frac{\text { Available energy }- \text { Arousal energy }}{\text { Arousal resistivity }}}
$$

Next, by using equation (21), two states can be compared. In the first state, the requested energy is high. In the second state, the requested energy is low.

$$
\begin{aligned}
\text { Arousal internal flux } 1 & =\sqrt{\frac{\text { Available energy }- \text { Arousal energy }}{\text { Aroush }}} \\
\text { Arousal interistivity } & \sqrt{\frac{\text { Available energy }- \text { Arousal energy } \text { flux }_{2}}{\text { Arousal resistivity }}} \\
\text { Ratio } & =\frac{\text { Arousal internal flux } 1}{\text { Arousal internal flux } 2} \Rightarrow \text { Ratio }<1
\end{aligned}
$$


The ratio will be lower than 1 . Using the signification of the internal flux ratio, a time dilation will be present and the time will be perceived slower (i.e. remembering more images during frightening situation).

The internal flux and external flux of the internal clock might regulate other mechanisms. These mechanisms would synchronize with these fluxes and play roles in the time perception chain of functions. For example, a visual recording function could be synchronized to the external flux, thereby recording the images at the same rate as the external flux, implying more images recorded in memory. Other functions not able to cope with a dangerous situation could synchronize on the internal flux to slow down their processes and liberate energy for the arousal mechanism or for other mechanisms that could deal with a dangerous situation.

\subsection{Attention mechanism}

Following the analogy of an operating system scheduler described in section 3.1, a supposition is made that the attention mechanism would trigger a normal priority process to request energy when needed. This process would be requested consciously during a meditation or less consciously during a walk in nature or a relaxing situation. The priority for the attention mechanism is estimated to be lower than the arousal mechanism. When attention would be needed, requested energy would be accumulated into an accumulator following the same pattern as the arousal mechanism. The energy requested and accumulated will be called the Attention energy. The Attention energy might also be used to send more pulses, record more images or call other brain functions. To represent the energy requested by the attention mechanism, equation (25) is used.

$$
\text { Attention external flux }=\sqrt{\frac{\text { Attention energy }}{\text { Attention resistivity }}}
$$

Next, by using equation (26), two states can be compared. In the first state, the requested energy is high. In the second state, the requested energy is low.

$$
\begin{aligned}
\text { Attention external flux } 1 & =\sqrt{\frac{\text { Attention energy }_{\text {high }}}{\text { Attention resistivity }}} \\
\text { Attention external flux } 2 & =\sqrt{\frac{\text { Attention energy low }}{\text { Attention resistivity }}} \\
\text { Ratio } & =\frac{\text { Attention external flux } 1}{\text { Attention external flux } 2} \Rightarrow \text { Ratio }>1
\end{aligned}
$$


The ratio will be higher than 1 . Using the signification of the clock external flux from section 2.9, the internal clock will tick faster when the attention is high. However, the person will perceive the time longer when the attention increases. To represent this perception, the signification of the clock internal flux section 2.9 is used.

$$
\text { Attention internal flux }=\sqrt{\frac{\text { Available energy }- \text { Attention energy }}{\text { Attention resistivity }}}
$$

Next, by using equation (21), two states can be compared. In the first state, the requested energy is high. In the second state, the requested energy is low.

$$
\begin{aligned}
\text { Attention internal flux } 1 & =\sqrt{\frac{\text { Available energy }- \text { Attention energy } y_{\text {high }}}{\text { Attention resistivity }}} \\
\text { Attention internal flux } 2 & =\sqrt{\frac{\text { Available energy }- \text { Attention energy low }}{\text { Attention resistivity }}} \\
\text { Ratio } & =\frac{\text { Attention internal flux } 1}{\text { Attention internal flux } 2} \Rightarrow \text { Ratio }<1
\end{aligned}
$$

The ratio will be lower than 1 . Using the signification of the internal flux ratio, a time dilation will be present and the time will be perceived slower.

\subsection{Specific attention mechanism}

When a task needs specific attention like calculations, the energy would be provided by the Attention energy. If no task needs attention, then the Attention energy would stay high and available until needed or until another mechanism, like the arousal mechanism, would request energy. If the arousal mechanism would request energy, the Attention energy would be reallocated in order to be available for the arousal mechanism. To represent a specific task requesting and consuming the Attention energy, a Task energy is subtracted from the Attention energy.

$$
\text { Attention external flux }=\sqrt{\frac{\text { Attention energy }- \text { Task energy }}{\text { Attention resistivity }}}
$$

Next, by using equation (26), two states can be compared. In the first state, the requested task energy is high. In the second state, the requested task energy is low.

$$
\begin{aligned}
\text { Attention external flux } 1 & =\sqrt{\frac{\text { Attention energy }- \text { Task energy } y_{\text {high }}}{\text { Attention resistivity }}} \\
\text { Attention external flux } 2 & =\sqrt{\frac{\text { Attention energy }- \text { Task energy } y_{\text {low }}}{\text { Attention resistivity }}} \\
\text { Ratio } & =\frac{\text { Attention external flux } 1}{\text { Attention external flux } 2} \Rightarrow \text { Ratio }<1
\end{aligned}
$$


The ratio will be lower than 1 as opposed to the ratio found in equation (42). The external flux of the internal clock will decelerate when a specific task requires higher energy. The attention internal flux would be represented by the following equation.

$$
\text { Attention internal flux }=\sqrt{\frac{\text { Available energy }-(\text { Attention energy }- \text { Task energy })}{\text { Attention resistivity }}}
$$

Next, by using equation (21), two states can be compared. In the first state, the requested task energy is high. In the second state, the requested task energy is low.

$$
\begin{aligned}
& \text { Attention internal flux } 1= \\
& \sqrt{\frac{\text { Available energy }-(\text { Attention energy }- \text { Task energy }}{\text { Attention }} \text { resistivity }} \\
& \text { Attention internal flux } 2= \\
& \sqrt{\frac{\text { Available energy }-\left(\text { Attention energy }- \text { Task energy } y_{\text {low }}\right)}{\text { Attention resistivity }}} \\
& \text { Ratio }=\frac{\text { Attention internal flux }}{1} \text { Attention internal flux } 2 \text { Ratio }>1
\end{aligned}
$$

The ratio will be higher than 1 , which is opposite to the ratio found in (44). The internal flux will accelerate when a specific task would require higher energy. The person would perceive the time accelerated while doing a specific task.

This consolidates the observations. If the attention is available without too much distractions, then the time seems slower. However, if a specific task is involved while the attention is available, then the time seems faster. 


\subsection{Combination of arousal, attention and specific attention mechanisms}

The arousal, attention and specific attention mechanisms can be combined into one equation which can represent the internal flux and external flux of the internal clock.

$$
\begin{aligned}
& \text { External flux }=\sqrt{\frac{\text { Arousal energy }+(\text { Attention energy }- \text { Task energy })}{\text { Resistivity }}} \\
& \text { Internal flux }= \\
& \sqrt{\frac{\text { Available energy }-(\text { Arousal energy }+(\text { Attention energy }- \text { Task energy }))}{\text { Resistivity }}}
\end{aligned}
$$

As an example, by using equation (26), two states of the external flux of the internal clock can be compared.

$$
\begin{aligned}
& \text { External } \text { flux }_{1}=\sqrt{\frac{\text { Arousal energy }_{1}+\left(\text { Attention energy }_{1}-\text { Task energy }_{1}\right)}{\text { Resistivity }_{1}}}
\end{aligned}
$$

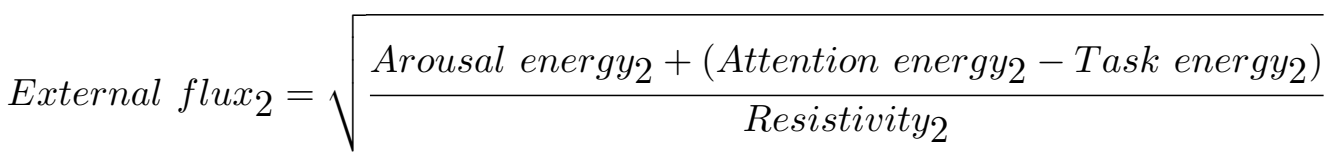

$$
\begin{aligned}
& \text { External flux ratio }=\frac{\text { External flux } 1}{\text { External flux }}
\end{aligned}
$$

The ratio satisfies the arousal and the attention mechanisms. More Arousal energy or more Attention energy in the External flux 1 will produce a ratio greater than 1, meaning the External flux 1 is faster, meaning the clock will be faster. However, if the Task energy is consuming more Attention energy in the External flux 1 , then the ratio might become less than 1, meaning that the External flux 1 might become slower, meaning the clock might become slower. The variation of the internal clock would be represented by a combination of these three parameters compared to their previous values.

To complete this section, an internal flux ratio is also represented. The ratio will have an inverse value of the ratio found in equation (51).

$$
\begin{aligned}
& \text { Internal } \text { flux }_{1}=
\end{aligned}
$$

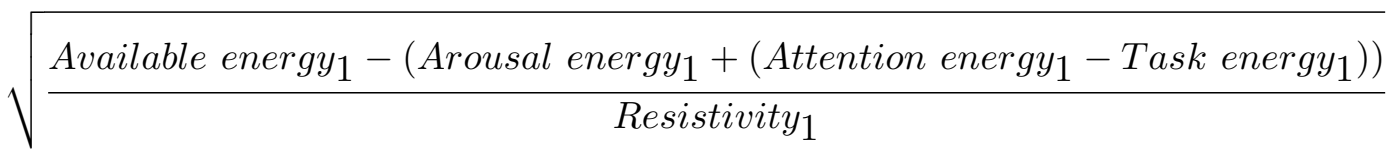

$$
\begin{aligned}
& \text { Internal } \text { flux }_{2}=
\end{aligned}
$$

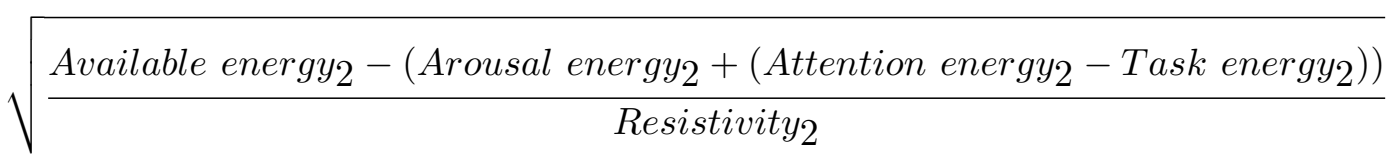

$$
\begin{aligned}
& \text { Internal flux ratio }=\frac{\text { Internal flux }}{\text { Internal flux }}
\end{aligned}
$$




\section{Combining physical time dilation and psychological time perception}

In this section, the transformation matrices are explained and used step by step in an example to combine physical time and psychological time perception.

\subsection{Parameters}

Four different situations are imagined (Situation A, Situation B, Situation $C$ and Situation D) in which four observers are considered (Subject A, Subject B, Experimenter $C$ and Experimenter $D$ ).

Subject A, Subject B and Experimenter $C$ are in a spaceship flying around the earth. Experimenter $D$ stayed on earth observing the spaceship.

\section{Situation A:}

Inside the spaceship, Subject $A(50 \mathrm{~kg})$ is driving an electric scooter $(20 \mathrm{~kg})$. The electric energy used to accelerate the scooter is $16800 \mathrm{~J}$. Subject $A$ has a flash light that automatically flashes at a speed of 2 flashes per second (2 flashes $/ s)$. Subject $A$ feels confident driving the scooter, the arousal is low. Subject $A$ feels relax and does not give attention to the task of driving the scooter, the attention task is low. Subject $_{A}$ has a high precision clock on the scooter to observe the elapsed time.

\section{Situation B:}

Inside the spaceship, Subject B $(100 \mathrm{~kg})$ is driving the same electric scooter model $(20 \mathrm{~kg})$. The electric energy used to accelerate the scooter is also $16800 \mathrm{~J}$. Subject $B$ has no flash light. Subject $B$ is frightened and does not feel confident driving the scooter, the arousal is high. Subject $B$ does not feel relax and gives much attention to the task of driving the scooter, the attention task is high. Subject $B$ has a high precision clock on the scooter to observe the elapsed time.

\section{Situation $C$ :}

Inside the spaceship that goes at the speed of $10000 \mathrm{~m} / \mathrm{s}$, Experimenter $C(50 \mathrm{~kg})$ is at rest seating on a seat, observing Subject $A$ and Subject B. Experimenter $C$ has a high precision clock to observe the elapsed time.

\section{Situation D:}

On earth, Experimenter $D(50 \mathrm{~kg})$ is at rest observing the spaceship in which Situation A, Situation $B$ and Situation $C$ are taking place. Experimenter $D$ has a high precision clock to observe the elapsed time. 
Each situation is considered an energy system. Each situation has an internal flux and an external flux. Fig. 2. represents the four situations. Experimenter $C$ is the main frame of reference of Subject $A$ and Subject B. Experimenter $D$ is the main frame of reference of Experimenter $C$ and indirectly can also be a main frame of reference of Subject $A$ and Subject $B$.

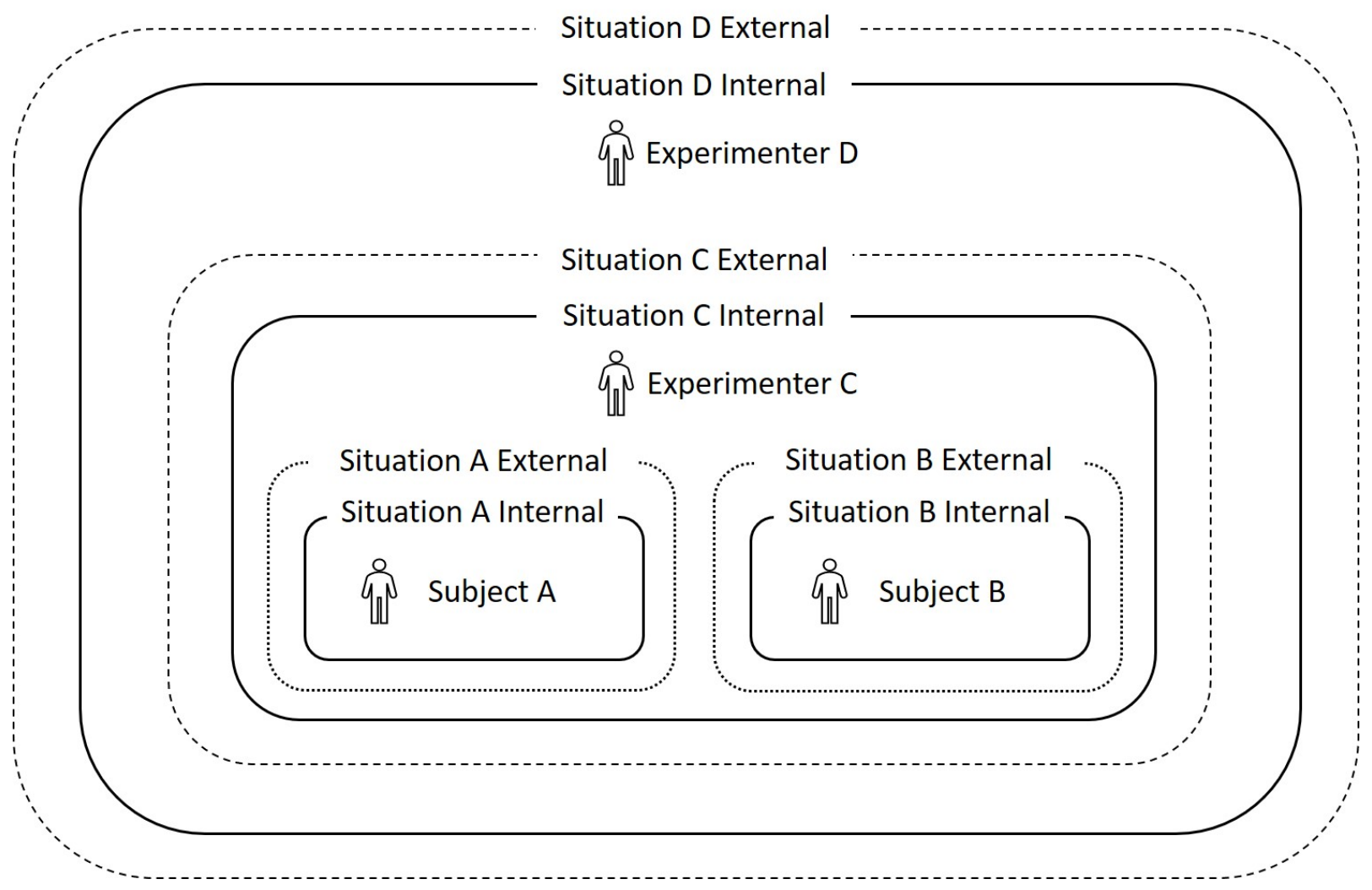

Fig. 2. Representation of the four situations

A first question is:

What will be the precise number of flashes that Subject $B$, Experimenter $C$ and Experimenter $D$ would perceive from Subject $A$ ?

A second question is:

What will be the speeds of Subject B clock, Experimenter $C$ clock and Experimenter D clock compared to Subject A clock?

Sections 4.2, 4.3 and 4.4 show the steps involved to find the answers. The result is presented in section 4.5. The interpretation of the result is presented in section 4.5.4. 


\subsection{Step 1 - Finding the internal and external fluxes}

The internal and external fluxes of the four situations are organized and presented using Table 1.

\section{Table 1}

Internal and external fluxes for situations $\mathrm{A}, \mathrm{B}, \mathrm{C}$ and $\mathrm{D}$

\begin{tabular}{c|c|c|c|c} 
Situation & Internal flux & External flux & Reference & Calculated time \\
\hline$A \mid C$ & $A_{I} \mid C$ & $A_{E} \mid C$ & $C$ & Physical \\
$A^{p} \mid C$ & $A_{I}^{p} \mid C$ & $A_{E}^{p} \mid C$ & $\mathrm{C}$ & Psychological \\
$B \mid C$ & $B_{I} \mid C$ & $B_{E}^{\mid C}$ & $C$ & Physical \\
$B^{p} \mid C$ & $B_{I}^{p} \mid C$ & $B_{E}^{p} \mid C$ & $C$ & Psychological \\
$C \mid D$ & $C_{I} \mid D$ & $C_{E}^{\mid D}$ & $D$ & Physical \\
$C \mid C$ & $C_{I} \mid C$ & $C_{E} \mid C$ & $C$ & Physical \\
$D \mid D$ & $D_{I} \mid D$ & $D_{E}^{\mid D}$ & $D$ & Physical
\end{tabular}

The bar sign, like in $A \mid C$, means that $C$ is the reference observer. It means that the values of Subject $A$ are dependent on Experimenter $C$ observation. The subscript $I$ like in $A_{I} \mid C$ stands for the Internal flux of Situation $A \mid C$. The subscript $E$ like in $A_{E} \mid C$ stands for the External flux of Situation $A \mid C$. The situations that have a superscript $p$ consider and calculate only the psychological time. The situations without the superscript $p$ consider the physical time (e.g. relativistic effect while in motion). The psychological time takes into account the arousal and attention mechanisms and is calculated using equation (49). The situations that are dependent on themselves, like $C \mid C$, are considered isolated, they do not interact with their environment, no Acting energy will be calculated.

The internal fluxes of situations that calculate the physical time are found using equation (20). Their external fluxes are found using equation (27).

The external fluxes of situations that calculate the psychological time are found using equation (49). Their internal fluxes are found using equation (27). 
Situation $A \mid C$ :

The following equations will be used by Experimenter $C$ to calculate the internal and external fluxes of Situation $A \mid C$.

$$
\begin{aligned}
& A_{I} \mid C=\sqrt{\frac{\left(M_{\text {scooter }}+M_{\text {Subject } A}\right) c^{2}-\text { Acting energy }}{M_{\text {scooter }}+M_{\text {Subject } A}}} \\
& A_{E} \mid C=\sqrt{c^{2}-\left(A_{I} \mid C\right)^{2}}
\end{aligned}
$$

Situation $A^{p} \mid C$ :

Due to the influence of the arousal and attention mechanisms on Subject A, a psychological time has to be calculated. Because the real energy consumed by the arousal and attention mechanisms are not known, a fictional energy consumption will be suggested. 10 percent of the resource will be shared between the arousal and the attention mechanisms. A mass of $1 \mathrm{~kg}$ of energy resource will be considered. The arousal will be low and estimated to be 1 percent of the 10 percent available energy. Then, 9 percent will be available for the attention mechanisms from which 2 percent will be used by the attention task to drive. The following equations will be used by Experimenter ${ }_{C}$ to calculate the internal and external fluxes of Situation $A^{p} \mid C$.

$$
\begin{aligned}
A_{E}^{p} \mid C & =\sqrt{\left.\frac{\text { Arousal energy }_{\text {low }}+(\text { Attention energy }- \text { Task energy }}{\text { Resistivity }}\right)} \\
& =\sqrt{\frac{1 \% \text { mass energy }+(9 \% \text { mass energy }-2 \% \text { mass energy })}{\text { mass }}} \\
A_{I}^{p} \mid C & =\sqrt{c^{2}-\left(A_{E}^{p} \mid C\right)^{2}}
\end{aligned}
$$

\section{Situation $B \mid C$ :}

The following equations will be used by Experimenter $C$ to calculate the internal and external fluxes of Situation $B \mid C$.

$$
\begin{aligned}
& B_{I} \mid C=\sqrt{\frac{\left(M_{\text {scooter }}+M_{\text {Subject } B}\right) c^{2}-\text { Acting energy }}{M_{\text {scooter }}+M_{\text {Subject } B}}} \\
& B_{E} \mid C=\sqrt{c^{2}-\left(B_{I} \mid C\right)^{2}}
\end{aligned}
$$

Situation $B^{p} \mid C$ :

Due to the influence of the arousal and attention mechanisms on Subject B, a psychological time has to be calculated. 10 percent of the resource will be shared between the arousal and the attention mechanisms. A mass of $1 \mathrm{~kg}$ of energy resource will be considered. Because Subject $B$ takes more care of the driving task, two times more attention energy will be allocated for the task. Because Subject $B$ is frightened, the arousal will be high and estimated to be 6 percent of the 10 percent available energy. 4 percent will be required 
for the attention mechanism from which 4 percent will be used by the attention task to drive, which is 2 times more than Subject $A$. The following equations will be used by Experiment $_{C}$ to calculate the internal and external fluxes of Situation $B^{p} \mid C$.

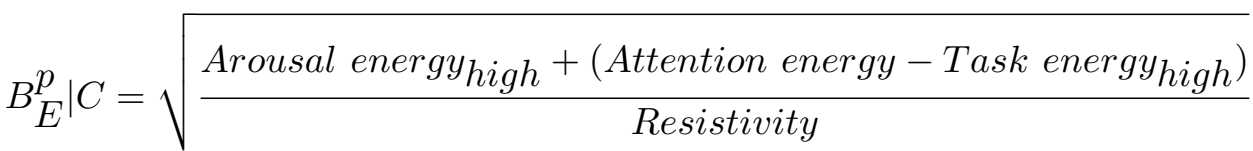

$$
\begin{aligned}
& =\sqrt{\frac{6 \% \text { mass energy }+(4 \% \text { mass energy }-4 \% \text { mass energy })}{\text { mass }}} \\
& B_{I}^{p} \mid C=\sqrt{c^{2}-\left(B_{E}^{p} \mid C\right)^{2}}
\end{aligned}
$$

Situation $C \mid D$ :

Experimenter $D$ will observe Experimenter $C$ seated within the spaceship in motion. To find $C_{I} \mid D$, Experimenter $D$ will apply to Situation $C \mid D$ an Acting energy of $M v^{2}$. We will not consider other Acting energy like gravity to not complicate the result.

$$
\begin{aligned}
& C_{I} \mid D=\sqrt{\frac{\left(M_{\text {Experimenter } C}\right) c^{2}-M v^{2}}{M_{\text {Experimenter } C}}} \\
& C_{E} \mid D=\sqrt{c^{2}-\left(C_{I} \mid D\right)^{2}}
\end{aligned}
$$

\section{Situation $C \mid C$ :}

$C_{I} \mid C$ will be calculated from Experimenter $C$ own point of view. Due to its isolation, there will be no Acting energy. The Internal flux will be maximal, equal to $c$. The External flux will be minimal, equal to zero.

$$
\begin{aligned}
C_{I} \mid C & =\sqrt{\frac{\left(M_{\text {Experimenter } C} c^{2}\right.}{M_{\text {Experimenter } C}}}=c \\
C_{E} \mid C & =\sqrt{c^{2}-\left(C_{I} \mid C\right)^{2}}=0
\end{aligned}
$$

\section{Situation $D \mid D$ :}

$D_{I} \mid C$ will be calculated from Experimenter $D$ own point of view. Due to its isolation, there will be no Acting energy. The Internal flux will be maximal, equal to $c$. The External flux will be minimal, equal to zero.

$$
\begin{aligned}
& D_{I} \mid D=\sqrt{\frac{\left(M_{\text {Experimenter } D}\right)^{2}}{M_{\text {Experimenter } D}}}=c \\
& D_{E} \mid D=\sqrt{c^{2}-\left(D_{I} \mid D\right)^{2}}=0
\end{aligned}
$$




\subsection{Step 2-Generating the transformation matrices}

The internal and external fluxes found in Step 1 can be combined to generate many ratios. As seen in equation (21) and (26), the ratios represent a dilation between two states which can be interpreted as a time dilation. The ratios found in Step 1 can be organized in tables. A table correspond to one situation fluxes over another situation fluxes. The tables needed to answer the questions are listed and described in Appendix A.2. To create the transformation matrices, the five following ratios will be used (found in tables A.3 to A.7).

$$
\frac{B_{I}}{A_{I}}\left|C \quad \frac{C_{I}}{A_{I}}\right| C \quad \frac{D_{I}}{C_{I}}\left|D \quad \frac{A_{I}^{p}}{A_{I}}\right| C \quad \frac{B_{I}^{p}}{B_{I}} \mid C
$$

The vertical bar is a short cut to signify that both the numerator and denominator calculations depend on the experimenter written after the vertical bar.

\subsubsection{Matrix $\left(A_{I} \rightarrow B_{I}\right) \mid C$, Subject A time to Subject B time, Experimenter $C$ view}

To compare the clock speed of Subject $A$ to the clock speed of Subject B, Experimenter $C$ must take the ratio $B_{I} / A_{I}$ of Table A.3. This ratio will be put in the first row and first column of a two by two matrix. The inverse of this ratio will be put into the last row and last column of the same matrix.

$$
\operatorname{Matrix}_{\left(A_{I} \rightarrow B_{I}\right) \mid C}=\left[\begin{array}{cc}
\frac{B_{I}}{A_{I}} \mid C & 0 \\
0 & \frac{A_{I}}{B_{I}} \mid C
\end{array}\right]
$$

This matrix transforms the time perceived by Subject $A$ clock into the time perceived by Subject $B$ clock, from the point of view of Experimenter $C$. It is Experimenter $C$ that will observe the scene, calculate the ratios and use the matrix.

To transform from Subject $A$ clock time to Subject $B$ clock time, a vector $V_{\text {ector }} A$ must be filled with two parameters. The first parameter is an elapsed time perceived by Subject A clock inside Situation A. The second parameter is a value representing a number of actions that Subject $A$ perceived on its scooter inside Situation A. An action can be the number of flashes made per second, the blood flow sent to its brain per second, a distance made per second by its arm moving or everything that can be compared to one second inside Situation $A$ (while on the scooter). Matrix $\left(A_{I} \rightarrow B_{I}\right) \mid C$ is then multiplied by this 
$\operatorname{Vector}_{A}$ to obtain a transformed Vector $_{B}$.

$$
\begin{gathered}
\text { Matrix }_{\left(A_{I} \rightarrow B_{I}\right) \mid C^{* \text { Vector }_{A}}}=\text { Vector }_{B} \\
{\left[\begin{array}{cc}
\frac{B_{I}}{A_{I}} \mid C & 0 \\
0 & \frac{A_{I}}{B_{I}} \mid C
\end{array}\right]\left[\begin{array}{c}
\text { seconds }_{A} \\
\text { actions }_{A} / \text { second }_{A}
\end{array}\right]}
\end{gathered}
$$

On the left side of the equal sign are the values obtained by Subject A inside Situation A, which are multiplied by the matrix. On the right side are the values transformed into Situation $B$ which will be perceived by Subject $B$. We use column-wise multiplication, meaning we put the matrix on the left side of vector $A$.

Example:

$$
\left[\begin{array}{ll}
2 & 0 \\
0 & \frac{1}{2}
\end{array}\right]\left[\begin{array}{c}
2 \text { seconds }_{A} \\
4 \text { actions }_{A} / \text { second }_{A}
\end{array}\right]=\left[\begin{array}{c}
4 \text { seconds }_{B} \\
2 \text { actions }_{A} / \text { second }_{B}
\end{array}\right]
$$

In this example, from the point of view of Experimenter $C$, if Subject $A$ clock is started at the same time as Subject B clock, then when 2 seconds will have elapsed on Subject $A$ clock, 4 seconds will have elapsed on Subject B clock. Subject B clock is two times faster than Subject $A$ clock. While 4 actions/second is made in Situation $A$ using Subject $A$ clock, only 2 actions of Situation $A$ will be achieved when 1 second of Subject $B$ clock has elapsed. When 2 seconds has elapsed with Subject $A$ clock, then 8 actions will be achieved in Situation A. However, when 8 actions in Situation $A$ is achieved then 4 seconds will have already elapsed on Subject $B$ clock.

A clarification must be made. In reality, Subject $B$ will not perceived visually the time inside Situation A. For this to happen, light has to travel from Situation $A$ to inside Situation $B$ so that Subject $B$ will be able to see the scene coming from Situation $A$. These imply a geometrical aspect between the two scenes. The variability between the path must be taken into account from the moment the light leaves Situation $A$ to arrive at Situation $B$ because both situations are in motion. General Relativity is a better tool to calculate this path (Einstein, 1916). The only postulate that can be made is that if a clock was to be used inside Situation $A$ and calibrated with the time from inside Situation $B$, then using this clock when calculating the actions inside Situation $A$ would give the result found in the right vector $\left(\operatorname{seconds}_{B}\right.$, actions $A /$ second $\left._{B}\right)$.

Using Matrix $\left(A_{I} \rightarrow B_{I}\right) \mid C$ and the matrices described in sections 4.3 .3 to 4.3.6, it will be possible to answer the questions. Matrix $\left(A_{I} \rightarrow B_{I}\right) \mid C$ will convert one second and the number of flashes per second from Subject $A$ to Subject $B$, from the point of view of Experimenter $C$. 


\subsubsection{Matrix $\left(A_{I} \rightarrow C_{I}\right) \mid C$, Subject A to Experimenter $C$, Experimenter $C$ view}

To convert from Subject $A$ time into Experimenter $C$ time, from Experimenter $C$ point of view, a matrix must be built using the ratio $C_{I} / A_{I}$ from Table A.4.

$$
\operatorname{Matrix}_{\left(A_{I} \rightarrow C_{I}\right) \mid C}=\left[\begin{array}{cc}
\frac{C_{I}}{A_{I}} \mid C & 0 \\
0 & \frac{A_{I}}{C_{I}} \mid C
\end{array}\right]
$$

\subsubsection{Matrix $\left(A_{I} \rightarrow D_{I}\right) \mid D$, Subject A to Experimenter D, Experimenter D view}

To convert from Subject A time into Experimenter $D$ time, from the point of view of Experimenter $D$, a matrix must be built using ratio $D_{I} / C_{I}$ from Table A.5 and ratio $C_{I} / A_{I}$ from Table A.4. Two ratios are needed because a direct transformation will not be correct. Indeed, Situation $A$ has been calculated using Experimenter $C$ point of view. We must then take into account an intermediate transformation from Experimenter $C$ time into Experimenter $D$ time, from Experimenter $D$ point of view.

$$
\operatorname{Matrix}_{\left(C_{I} \rightarrow D_{I}\right) \mid D}=\left[\begin{array}{cc}
\frac{D_{I}}{C_{I}} \mid D & 0 \\
0 & \frac{C_{I}}{D_{I}} \mid D
\end{array}\right]
$$

$\operatorname{Matrix}_{\left(C_{I} \rightarrow D_{I}\right) \mid D}$ is multiplied by Matrix $\left(A_{I} \rightarrow C_{I}\right) \mid C$ to obtain Matrix $\left(A_{I} \rightarrow D_{I}\right) \mid D$.

$$
\begin{aligned}
\text { Matrix }_{\left(A_{I} \rightarrow D_{I}\right) \mid D} & =\left[\begin{array}{cc}
\frac{D_{I}}{C_{I}} \mid D & 0 \\
0 & \frac{C_{I}}{D_{I}} \mid D
\end{array}\right]\left[\begin{array}{cc}
\frac{C_{I}}{A_{I}} \mid C & 0 \\
0 & \frac{A_{I}}{C_{I}} \mid C
\end{array}\right] \\
& =\left[\begin{array}{cc}
\frac{D_{I}}{C_{I}}\left|D * \frac{C_{I}}{A_{I}}\right| C & 0 \\
0 & \frac{C_{I}}{D_{I}}\left|D * \frac{A_{I}}{C_{I}}\right| C
\end{array}\right]
\end{aligned}
$$

\footnotetext{
4.3.4. Matrix $\left(A_{I} \rightarrow A_{I}^{p}\right) \mid C$, Subject A physical time to Subject A psychological time, Experimenter $C$ view
}

To convert from Subject A physical time into Subject $A$ psychological time, a matrix must be built using ratio $A_{I}^{p} / A_{I}$ from Table A.6.

$$
\operatorname{Matrix}_{\left(A_{I} \rightarrow A_{I}^{p}\right) \mid C}=\left[\begin{array}{cc}
\frac{A_{I}^{p}}{A_{I}} \mid C & 0 \\
0 & \frac{A_{I}}{A_{I}^{p}} \mid C
\end{array}\right]
$$




\subsubsection{Matrix $\left(B_{I} \rightarrow B_{I}^{p}\right) \mid C$, Subject B physical time to Subject B psychological time, Experimenter $C$ view}

To convert from Subject $B$ physical time into Subject $B$ psychological time, a matrix must be built using ratio $B_{I}^{p} / B_{I}$ from Table A.7.

$$
\operatorname{Matrix}_{\left(B_{I} \rightarrow B_{I}^{p}\right) \mid C}=\left[\begin{array}{cc}
\frac{B_{I}^{p}}{B_{I}} \mid C & 0 \\
0 & \frac{B_{I}}{B_{I}^{p}} \mid C
\end{array}\right]
$$

4.3.6. Matrix $\left(A_{I} \rightarrow B_{I}^{p}\right) \mid C$, Subject A physical time to Subject B psychological time, Experimenter $C$ view

To convert from Subject $A$ physical time into Subject $B$ psychological time, a matrix must be built using Matrix $\left(A_{I} \rightarrow B_{I}\right) \mid C$ and Matrix ${ }_{\left(B_{I} \rightarrow B_{I}^{p}\right) \mid C}$.

However, because they have the same reference observer $\left(\right.$ Experimenter $\left._{C}\right)$, we could have also created directly Matrix $\left(A_{I} \rightarrow B_{I}^{p}\right) \mid C$ using the ratio $B_{I}^{p} / A_{I}$.

$$
\operatorname{Matrix}_{\left(A_{I} \rightarrow B_{I}^{p}\right) \mid C}=\left[\begin{array}{cc}
\frac{B_{I}}{A_{I}} \mid C & 0 \\
0 & \frac{A_{I}}{B_{I}} \mid C
\end{array}\right]\left[\begin{array}{cc}
\frac{B_{I}^{p}}{B_{I}} \mid C & 0 \\
0 & \frac{B_{I}}{B_{I}^{p}} \mid C
\end{array}\right]=\left[\begin{array}{cc}
\frac{B_{I}^{p}}{A_{I}} \mid C & 0 \\
0 & \frac{A_{I}}{B_{I}^{p}} \mid C
\end{array}\right]
$$




\subsection{Step 3-Applying the transformations}

To apply the transformations, each matrix of step 2 must be multiplied by a vector representing Subject A parameters. The parameters are a duration in seconds and a number of flashes per second.

$$
\text { Subject A vector }=\left[\begin{array}{c}
\text { seconds }_{A} \\
\text { flashes }_{A} / \text { second }_{A}
\end{array}\right]
$$

4.4.1. Subject A to Subject B using Matrix $\left(A_{I} \rightarrow B_{I}\right) \mid C$

$$
\left[\begin{array}{cc}
\frac{B_{I}}{A_{I}} \mid C & 0 \\
0 & \frac{A_{I}}{B_{I}} \mid C
\end{array}\right]\left[\begin{array}{c}
\text { seconds }_{A} \\
\text { flashes }_{A} / \text { second }_{A}
\end{array}\right]=\left[\begin{array}{c}
\text { seconds }_{B} \\
\text { flashes }_{A} / \text { second }_{B}
\end{array}\right]
$$

4.4.2. Subject $A$ to Experimenter $C$ using Matrix $\left(A_{I} \rightarrow C_{I}\right) \mid C$

$$
\left[\begin{array}{cc}
\frac{C_{I}}{A_{I}} \mid C & 0 \\
0 & \frac{A_{I}}{C_{I}} \mid C
\end{array}\right]\left[\begin{array}{c}
\text { seconds }_{A} \\
\text { flashes }_{A} / \text { second }_{A}
\end{array}\right]=\left[\begin{array}{c}
\text { seconds }_{C} \\
\text { flashes }_{A} / \text { second }_{C}
\end{array}\right]
$$

4.4.3. Subject $A$ to Experimenter $D$ using Matrix $\left(A_{I} \rightarrow D_{I}\right) \mid D$

$$
\left[\begin{array}{cc}
\frac{D_{I}}{C_{I}}\left|D * \frac{C_{I}}{A_{I}}\right| C & 0 \\
0 & \frac{C_{I}}{D_{I}}\left|D * \frac{A_{I}}{C_{I}}\right| C
\end{array}\right]\left[\begin{array}{c}
\text { seconds } A_{\text {flashes }_{A} / \text { second }_{A}}
\end{array}\right]=\left[\begin{array}{c}
\text { seconds }_{D} \\
\text { flashes }_{A} / \text { second }_{D}
\end{array}\right]
$$

\subsubsection{Subject A physical time to Subject A psychological time} using Matrix $\left(A_{I} \rightarrow A_{I}^{p}\right) \mid C$

$$
\left[\begin{array}{cc}
\frac{A_{I}^{p}}{A_{I}} \mid C & 0 \\
0 & \frac{A_{I}}{A_{I}^{p}} \mid C
\end{array}\right]\left[\begin{array}{c}
\text { seconds } A_{\text {flashes }_{A} / \text { second }_{A}}
\end{array}\right]=\left[\begin{array}{c}
\text { seconds }_{A} \\
\text { flashes }_{A} / \text { second }_{A}
\end{array}\right]
$$


4.4.5. Subject B physical time to Subject B psychological time using Matrix $\left(B_{I} \rightarrow B_{I}^{p}\right) \mid C$

$$
\left[\begin{array}{cc}
\frac{B_{I}^{p}}{B_{I}} \mid C & 0 \\
0 & \frac{B_{I}}{B_{I}^{p}} \mid C
\end{array}\right]\left[\begin{array}{c}
\text { seconds }_{B} \\
\text { flashes }_{B} / \text { second }_{B}
\end{array}\right]=\left[\begin{array}{c}
\text { seconds }_{B} \\
\text { flashes }_{B} / \text { second }_{B}
\end{array}\right]
$$

4.4.6. Subject A physical time to Subject B psychological time using Matrix $\left(A_{I} \rightarrow B_{I}^{p}\right) \mid C$

$$
\left[\begin{array}{cc}
\frac{B_{I}^{p}}{A_{I}} \mid C & 0 \\
0 & \frac{A_{I}}{B_{I}^{p}} \mid C
\end{array}\right]\left[\begin{array}{c}
\text { seconds }_{A} \\
\text { flashes }_{A} / \text { second }_{A}
\end{array}\right]=\left[\begin{array}{c}
\text { seconds }_{B} p \\
\text { flashes }_{A} / \text { second }_{B}
\end{array}\right]
$$




\subsection{Results}

The results of each step are presented in this section.

\subsubsection{Result step 1}

Table 2 shows the result calculated in step 1. The intermediate calculations are found in Appendix A.1.

\section{Table 2}

Result step 1

\begin{tabular}{c|c|c} 
Situation & Internal flux & External flux \\
\hline$A \mid C$ & $A_{I} \mid C=299792457.9999996$ & $A_{E} \mid C=15.4919334$ \\
$A^{p} \mid C$ & $A_{I}^{p} \mid C=287550824.1055609$ & $A_{E}^{p} \mid C=84794112.0001533$ \\
$B \mid C$ & $B_{I} \mid C=299792457.9999998$ & $B_{E} \mid C=11.8321596$ \\
$B^{p} \mid C$ & $B_{I}^{p} \mid C=290659572.0103862$ & $B_{E}^{p} \mid C=73433855.0834757$ \\
$C \mid D$ & $C_{I} \mid D=299792457.8332180$ & $C_{E} \mid D=10000$ \\
$C \mid C$ & $C_{I} \mid C=299792458$ & $C_{E} \mid C=0$ \\
$D \mid D$ & $D_{I} \mid D=299792458$ & $D_{E} \mid D=0$
\end{tabular}

\subsubsection{Result step 2}

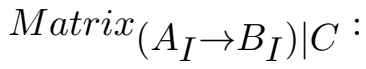

$$
\left[\begin{array}{cc}
\frac{B_{I}}{A_{I}} \mid C & 0 \\
0 & \frac{A_{I}}{B_{I}} \mid C
\end{array}\right]=\left[\begin{array}{cc}
1.00000000000000067 & 0 \\
0 & 0.99999999999999944
\end{array}\right]
$$

Matrix $\left(A_{I} \rightarrow C_{I}\right) \mid C$ :

$$
\left[\begin{array}{cc}
\frac{C_{I}}{A_{I}} \mid C & 0 \\
0 & \frac{A_{I}}{C_{I}} \mid C
\end{array}\right]=\left[\begin{array}{cc}
1.00000000000000133 & 0 \\
0 & 0.99999999999999867
\end{array}\right]
$$


$\operatorname{Matrix}_{\left(C_{I} \rightarrow D_{I}\right) \mid D}$ : $^{-}$

$$
\left[\begin{array}{cc}
\frac{D_{I}}{C_{I}} \mid D & 0 \\
0 & \frac{C_{I}}{D_{I}} \mid D
\end{array}\right]=\left[\begin{array}{cc}
1.00000000055632499 & 0 \\
0 & 0.99999999944367501
\end{array}\right]
$$

$\operatorname{Matrix}_{\left(A_{I} \rightarrow D_{I}\right) \mid D}:$

$$
\left[\begin{array}{cc}
\frac{D_{I}}{C_{I}}\left|D * \frac{C_{I}}{A_{I}}\right| C & 0 \\
0 & \frac{C_{I}}{D_{I}}\left|D * \frac{A_{I}}{C_{I}}\right| C
\end{array}\right]=\left[\begin{array}{cc}
1.00000000055632632 & 0 \\
0 & 0.99999999944367368
\end{array}\right]
$$

$\operatorname{Matrix}_{\left(A_{I} \rightarrow A_{I}^{p}\right) \mid C}:$

$$
\left[\begin{array}{cc}
\frac{A_{I}^{p}}{A_{I}} \mid C & 0 \\
0 & \frac{A_{I}}{A_{I}^{p}} \mid C
\end{array}\right]=\left[\begin{array}{cc}
0.95916630466254515 & 0 \\
0 & 1.04257207028537247
\end{array}\right]
$$

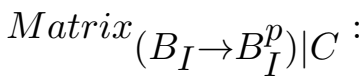

$$
\left[\begin{array}{cc}
\frac{B_{I}^{p}}{B_{I}} \mid C & 0 \\
0 & \frac{B_{I}}{B_{I}^{p}} \mid C
\end{array}\right]=\left[\begin{array}{cc}
0.96953597148326653 & 0 \\
0 & 1.03142124625879261
\end{array}\right]
$$

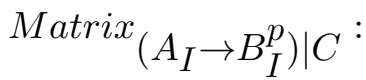

$$
\left[\begin{array}{cc}
\frac{B_{I}^{p}}{A_{I}} \mid C & 0 \\
0 & \frac{A_{I}}{B_{I}^{p}} \mid C
\end{array}\right]=\left[\begin{array}{cc}
0.96953597148326709 & 0 \\
0 & 1.03142124625879195
\end{array}\right]
$$




\subsubsection{Result step 3}

Subject $_{A}$ parameters:

$$
\operatorname{Vector}_{A}=\left[\begin{array}{c}
1 \text { second }_{A} \\
2 \text { flashes }_{A} / \text { second }_{A}
\end{array}\right]
$$

Subject A to Subject B (Experimenter $C$ view):

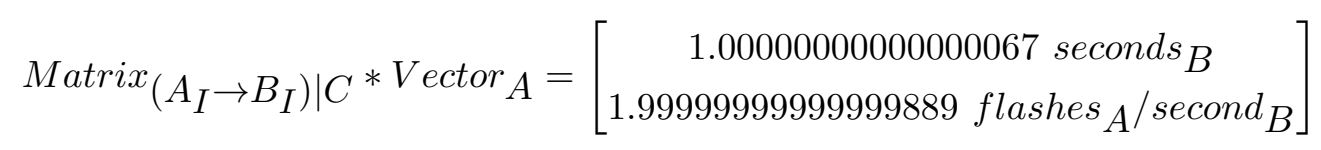

Subject A to Experimenter $C$ (Experimenter $C$ view):

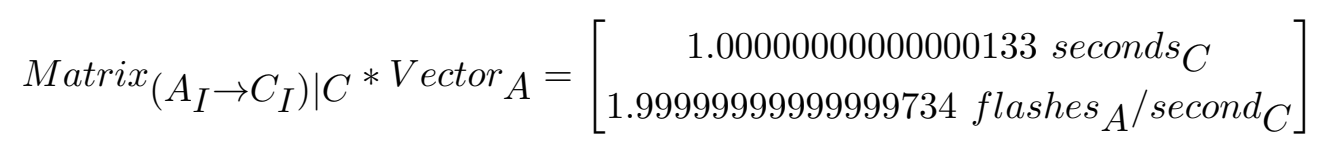

Subject $A$ to Experimenter $D$ (Experimenter D view):

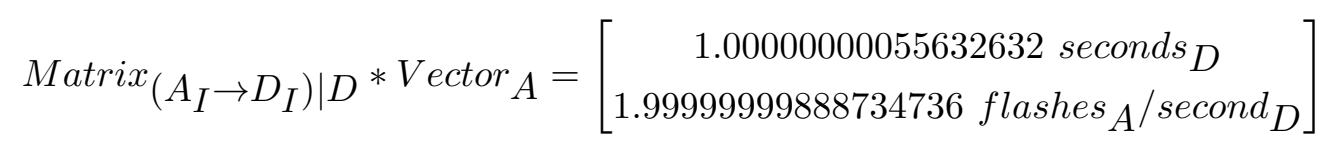

Subject A physical time to Subject A psychological time (Experimenter C view):

$$
\text { Matrix }_{\left(A_{I} \rightarrow A_{I}^{p}\right) \mid C} \text { Vector }_{A}=\left[\begin{array}{c}
0.95916630466254515 \text { seconds }_{A}^{p} \\
2.08514414057074493 \text { flashes }_{A} / \text { second }_{A} p
\end{array}\right]
$$

Subject A physical time to Subject B psychological time (Experimenter C view):

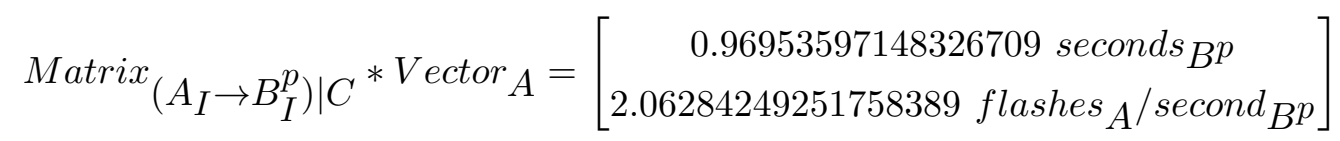




\subsubsection{Result interpretation}

The dilations are small because the speeds of the scooters and the spaceship are low compared to the speed of light. On earth, when motions are calculated, the influence of the relativistic effect is too low to be considered in the result. The dilation is significant when the speed approaches the speed of light.

Equation (85) shows that when 1 second has elapsed on Subject A clock then 1.00000000000000067 second has elapsed on Subject B clock. Subject B clock is faster than Subject A clock. When 1 second has elapsed on Subject A clock, 2 flashes will have occurred inside Situation A. However, when 1 second has elapsed on Subject B clock, only 1.99999999999999889 flashes will have occurred inside Situation A. Subject B clock will reach 1.00000000000000067 second before the 2 flashes occur inside Situation A. The same interpretation is applied in equations (86) and (87).

Equation (88) shows that when 1 second has elapsed on Subject $A$ clock then only $\approx 0.959$ second will be perceived by Subject $A$ brain. The time will be perceived as slowed down. Indeed, when 1 second will be perceived by Subject $A$ brain, then $\approx 2.085$ flashes will already have occurred inside Situation $A$. The perception is slower than the reality. The same interpretation is applied for equation (89). Subject $A$ brain and Subject $B$ brain will both perceive a time dilation. Subject $A$ mainly due to its higher attention energy and Subject $B$ mainly due to its higher arousal energy.

Looking at Table 2, the external flux $A_{E}^{p} \mid C$ is faster than the external flux $B_{E}^{p} \mid C$, which indicates that the brain's internal clock of $S u b j e c t ~ A$ is faster than the brain's internal clock of Subject B. Subject $A$ brain will perceive the time slower than Subject B brain mainly because Subject $B$ is given more attention to the task. 


\section{Conclusion}

Using the model, a connection between the relativistic view on time dilation and the psychological view on time perception was provided. The mechanism that binds both views is the use of energy ratios to represent time dilation. The more energy difference in a ratio, the more time dilation is present in both physical time and psychological time.

Brain mechanisms like the arousal and attention mechanisms were represented using relativistic equations in the form of internal flux ratios and external flux ratios. The transformation matrices are a tool provided to assist in the calculations of the time dilation between different observers, by including the physical time and psychological time.

Using the concept of fluxes, a combination of physical time dilation and psychological time perception was possible.

The presented model as well as the representation of brain mechanisms fluxes using this model and the transformation matrices are an approach to bring closer time dilation and time perception.

The model is a theoretical attempt and it might need improvement and more experimental observations to support and validate it.

The approach presented using the concept of energy ratio to express time dilation instead of geometrical transformations might also assist some people and outsiders in understanding better the subject of time dilation.

With today's possibility to travel from earth to other planets, it might be interesting to investigate more the relation between time dilation and time perception.

A perspective would be a discipline having time dilation as its central theme. Time dilation is mostly confined to physics and engineering disciplines. It can certainly be extend to other disciplines like the attempt made in this paper. 


\section{Appendix A. Calculations step 1 and flux ratio tables}

Appendix A.1. Intermediate calculations step 1

$A_{I} \mid C$ :

$$
\begin{aligned}
\text { Internal intensity } & =\frac{(20 \mathrm{~kg}+50 \mathrm{~kg}) \mathrm{c}^{2}-16800 \mathrm{~J}}{20 \mathrm{~kg}+50 \mathrm{~kg}}=c^{2}-\frac{240 \mathrm{~J}}{\mathrm{~kg}}=c^{2}-240 \mathrm{~m}^{2} \mathrm{~s}^{-2} \\
A_{I} \mid C & =\sqrt{c^{2}-240 \mathrm{~m}^{2} \mathrm{~s}^{-2}}=299792457.9999996 \mathrm{f}_{\mathrm{m} / \mathrm{s}}
\end{aligned}
$$

$A_{E} \mid C$ :

$$
\begin{aligned}
\text { External intensity } & =c^{2}-\left(c^{2}-240 m^{2} s^{-2}\right)=240 m^{2} s^{-2} \\
A_{E} \mid C & =\sqrt{240 m^{2} s^{-2}}=15.4919334 f_{m} / s
\end{aligned}
$$

$A_{E}^{p} \mid C$ :

$$
\begin{aligned}
\text { External intensity } & =\frac{0.01 * M c^{2}+\left(0.09 * M c^{2}-0.02 * M c^{2}\right)}{M}=0.08 * c^{2} \\
A_{E}^{p} \mid C & =\sqrt{0.08 * c^{2}}=84794112.0001533 f_{m / s}
\end{aligned}
$$

$A_{I}^{p} \mid C$

$$
A_{I}^{p} \mid C=\sqrt{c^{2}-\left(A_{E}^{p} \mid C\right)^{2}}=\sqrt{c^{2}-0.08 * c^{2}}=287550824.1055609 f_{\mathrm{m} / \mathrm{s}}
$$

$B_{I} \mid C$ :

$$
\begin{aligned}
\text { Internal intensity } & =\frac{(20 k g+100 k g) c^{2}-16800 J}{20 k g+100 k g}=c^{2}-\frac{140 J}{k g}=c^{2}-140 m^{2} s^{-2} \\
B_{I} \mid C & =\sqrt{c^{2}-140 m^{2} s^{-2}}=299792457.9999998 f_{m / s}
\end{aligned}
$$

$B_{E} \mid C$ :

$$
\begin{aligned}
\text { External intensity } & =c^{2}-\left(c^{2}-140 \mathrm{~m}^{2} s^{-2}\right)=140 \mathrm{~m}^{2} \mathrm{~s}^{-2} \\
B_{E} \mid C & =\sqrt{140 \mathrm{~m}^{2} \mathrm{~s}^{-2}}=11.8321596 \mathrm{fm} / \mathrm{s}
\end{aligned}
$$

$B_{E}^{p} \mid C$ :

$$
\begin{aligned}
\text { External intensity } & =\frac{0.06 * M c^{2}+\left(0.04 * M c^{2}-0.04 * M c^{2}\right)}{M}=0.06 * c^{2} \\
B_{E}^{p} \mid C & =\sqrt{0.06 * c^{2}}=73433855.0834757 \mathrm{f}_{\mathrm{m} / \mathrm{s}}
\end{aligned}
$$

$B_{I}^{p} \mid C$ :

$$
B_{I}^{p} \mid C=\sqrt{c^{2}-\left(B_{E}^{p} \mid C\right)^{2}}=\sqrt{c^{2}-0.06 * c^{2}}=290659572.0103862 f_{m / s}
$$


$C_{I} \mid D:$

$$
\begin{aligned}
\text { Internal intensity } & =\frac{(50 \mathrm{~kg}) \mathrm{c}^{2}-50 \mathrm{~kg} *(10000 \mathrm{~m} / \mathrm{s})^{2}}{50 \mathrm{~kg}}=c^{2}-100000000 \mathrm{~m}^{2} \mathrm{~s}^{-2} \\
C_{I} \mid D & =\sqrt{c^{2}-100000000 \mathrm{~m}^{2} \mathrm{~s}^{-2}}=299792457.8332180 \mathrm{f}_{\mathrm{m} / \mathrm{s}}
\end{aligned}
$$

$C_{E} \mid D:$

$$
\begin{aligned}
\text { External intensity } & =c^{2}-\left(c^{2}-100000000 \mathrm{~m}^{2} s^{-2}\right)=100000000 \mathrm{~m}^{2} s^{-2} \\
C_{E} \mid D & =\sqrt{100000000 \mathrm{~m}^{2} s^{-2}}=10000 f_{\mathrm{m} / \mathrm{s}}
\end{aligned}
$$

$C_{I} \mid C$ :

$$
\begin{aligned}
\text { Internal intensity } & =\frac{(50 \mathrm{~kg}) \mathrm{c}^{2}}{50 \mathrm{~kg}}=c^{2} \\
C_{I} \mid C & =\sqrt{c^{2}}=299792458 \mathrm{f}_{\mathrm{m} / \mathrm{s}}
\end{aligned}
$$

$C_{E} \mid C$ :

$$
\begin{aligned}
\text { External intensity } & =c^{2}-c^{2}=0 \\
C_{E} \mid C & =\sqrt{0}=0 f_{m / s}
\end{aligned}
$$

$D_{I} \mid D:$

$$
\begin{aligned}
\text { Internal intensity } & =\frac{(50 \mathrm{~kg}) \mathrm{c}^{2}}{50 \mathrm{~kg}}=c^{2} \\
D_{I} \mid D & =\sqrt{c^{2}}=299792458 \mathrm{f}_{\mathrm{m} / \mathrm{s}}
\end{aligned}
$$

$D_{E} \mid D:$

$$
\begin{aligned}
\text { External intensity } & =c^{2}-c^{2}=0 \\
D_{E} \mid D & =\sqrt{0}=0 f_{m / s}
\end{aligned}
$$


The internal flux ratios used in step 2 are found in Tables A.3, A.4, A.5, A.6 and A.7.

Table A.3

Table A.4

Situation $B \mid C$ over Situation $A \mid C$ Situation $C \mid C$ over Situation $A \mid C$ Situation $D \mid D$ over
Situation $C \mid D$

Table A.5

\begin{tabular}{l|l|l} 
& $C_{I} \mid C$ & $C_{E} \mid C$ \\
\hline$A_{I} \mid C$ & $\frac{C_{I}}{A_{I}} \mid C$ & $\frac{C_{E}}{A_{I}} \mid C$ \\
$A_{E} \mid C$ & $\frac{C_{I}}{A_{E}} \mid C$ & $\frac{C_{E}}{A_{E}} \mid C$
\end{tabular}

Table A.6

Situation $A^{p} \mid C$ over

Situation $A \mid C$

\begin{tabular}{c|c|c} 
& $A_{I}^{p} \mid C$ & $A_{E}^{p} \mid C$ \\
\hline$A_{I} \mid C$ & $\frac{A_{I}^{p}}{A_{I}} \mid C$ & $\frac{A_{E}^{p}}{A_{I}} \mid C$ \\
$A_{E} \mid C$ & $\frac{A_{I}^{p}}{A_{E}} \mid C$ & $\frac{A_{E}^{p}}{A_{E}} \mid C$
\end{tabular}

\section{Table A.7}

Situation $B^{p} \mid C$ over

Situation $B \mid C$

\section{Appendix A.2.1. Table creation}

To create mathematically a table of ratios, two vectors are needed. The first vector corresponds to the internal and external fluxes of one situation. The second vector corresponds to the the internal and external fluxes values of another situation, but inverted.

$$
\begin{aligned}
\text { First vector : Situation } B \mid C & =\left[\begin{array}{ll}
B_{I} \mid C & B_{E} \mid C
\end{array}\right] \\
\text { Second vector: Situation } A \mid C & =\left[\begin{array}{cc}
\frac{1}{A_{I} \mid C} & \frac{1}{A_{E} \mid C}
\end{array}\right]
\end{aligned}
$$


A matrix is obtained by multiplying the two vectors using an outer product operator $\otimes$. The matrix corresponds to Table A.3. Situation $A \mid C$ must be on the left side of the outer product operator.

Table $A .3=$ Situation $A \mid C \otimes$ Situation $B \mid C$

$$
\Rightarrow \text { Table } A .3=\left[\begin{array}{c}
\frac{1}{A_{I} \mid C} \\
\frac{1}{A_{E} \mid C}
\end{array}\right] \otimes\left[\begin{array}{ll}
B_{I} \mid C & B_{E} \mid C
\end{array}\right]=\left[\begin{array}{ll}
\frac{B_{I}}{A_{I}} \mid C & \frac{B_{E}}{A_{I}} \mid C \\
\frac{B_{I}}{A_{E}} \mid C & \frac{B_{E}}{A_{E}} \mid C
\end{array}\right]
$$

Appendix A.2.2. Ratio $\frac{B_{I}}{A_{I}} \mid C$

This ratio compares the two internal fluxes. It gives, from the point of view of Observer $C$, the time dilation between Observer $B$ clock and Observer $A$ clock. This ratio is described and used in section 4.3.

Appendix A.2.3. Ratio $\frac{B_{E}}{A_{E}} \mid C$

This ratio compares the two external fluxes. It gives, from the point of view of Observer $C$ clock, the difference (dilation) in speed between Observer $B$ external speed and Observer A external speed. A matrix can be created from this ratio.

$$
\operatorname{Matrix}\left(A_{E} \rightarrow B_{E}\right) \mid C=\left[\begin{array}{cc}
\frac{B_{E}}{A_{E}} \mid C & 0 \\
0 & \frac{A_{E}}{B_{E}} \mid C
\end{array}\right]
$$

If Observer $_{A}$ speed is $4 \mathrm{~m} / \mathrm{s}$ and Observer $_{B}$ speed is $8 \mathrm{~m} / \mathrm{s}$, the matrix will be the following values.

$$
\operatorname{Matrix}\left(A_{E} \rightarrow B_{E}\right) \mid C=\left[\begin{array}{cc}
8 & \\
\overline{4} \mid C & 0 \\
0 & \frac{4}{8} \mid C
\end{array}\right]=\left[\begin{array}{cc}
2 & 0 \\
0 & \frac{1}{2}
\end{array}\right]
$$

This matrix permits to compare the distance and the time (using Observer $C$ clock) to accomplish something using Situation $A$ and using Situation B. For example, if Situation A scooter reaches a distance of 12 meters, Situation $B$ scooter will have reached a distance of 24 meters (2 times more). Moreover, if Situation A scooter takes 6 seconds to achieve a certain distance, Situation $B$ scooter will take 3 seconds (2 times faster). However, this 
matrix does not give the speed of each scooter, but only compare their relative distance and time to accomplish something from Observer $C$ point of view:

$$
\left[\begin{array}{ll}
2 & 0 \\
0 & \frac{1}{2}
\end{array}\right]\left[\begin{array}{l}
12 \text { meter }_{A} \\
6 \text { seconds }_{A}
\end{array}\right]=\left[\begin{array}{l}
24 \text { meter } \\
B \\
3 \text { seconds } \\
B
\end{array}\right]
$$

Appendix A.2.4. Ratio $\frac{B_{E}}{A_{I}} \mid C$ and $\frac{B_{I}}{A_{E}} \mid C$

These ratios compare, from Observer $C$ point of view, the external/internal flux of one situation to the internal/external flux of another situation. These ratios are more complex to interpret. However, these ratios can take the same meaning as in Appendix A.2.3. For example, if Situation A external flux is $1000 \mathrm{~m} / \mathrm{s}$, then Situation A internal flux will be $\approx 299790790 \mathrm{~m} / \mathrm{s}$ and if Situation B external flux is $299785787 \mathrm{~m} / \mathrm{s}$, then Situation B internal flux will be $\approx 2000 \mathrm{~m} / \mathrm{s}$. We can create the two following matrices.

$$
\begin{aligned}
& \text { Matrix }\left(B_{E} \rightarrow A_{I}\right) \mid C=\left[\begin{array}{cc}
\frac{299790790}{299785787} & 0 \\
0 & \frac{299785787}{299790790}
\end{array}\right]=\left[\begin{array}{cc}
1.000017 & 0 \\
0 & 0.9999833
\end{array}\right] \\
& \text { Matrix }_{\left(A_{E} \rightarrow B_{I}\right) \mid C}=\left[\begin{array}{cc}
\frac{2000}{1000} & 0 \\
0 & \frac{1000}{2000}
\end{array}\right]=\left[\begin{array}{ll}
2 & 0 \\
0 & 1
\end{array}\right]
\end{aligned}
$$

Equation (A.20) shows that Situation $A$ internal flux is faster than Situation $B$ external flux. Equation (A.21) shows that Situation $B$ internal flux is faster than Situation $A$ external flux. 


\section{Appendix B. Correlation with the time dilation formulas}

Appendix B.1. Lorentz factor correlation

The chosen Acting energy representing a system in motion is $m v^{2}$. To compare two systems, one in motion and one at rest, the following equation is used.

$$
\text { Internal flux ratio }=\sqrt{\frac{\left(\frac{M c^{2}-M v^{2}}{M}\right)_{\text {motion }}}{\left(\frac{M c^{2}}{M}\right)_{\text {rest }}}}
$$

In the next example, two clocks are considered, one in motion and one at rest. Both clocks have the same mass. The clock in motion has a speed of $1000 \mathrm{~m} / \mathrm{s}$. The internal fluxes of both clocks are compared.

$$
\begin{aligned}
\text { Internal flux motion } & =\sqrt{\frac{M c^{2}-M *(1000 \mathrm{~m} / \mathrm{s})^{2}}{M}}=299792457.99833 f_{\mathrm{m} / \mathrm{s}} \\
\text { Internal flux rest } & =\sqrt{\frac{M c^{2}}{M}=299792458 f_{\mathrm{m} / \mathrm{s}}} \\
\text { Internal flux ratio } & =\sqrt{\frac{\text { Internal flux } \text { motion }}{\text { Internal flux rest }}}=0.999999999994 \\
\text { Internal flux ratio } & =\sqrt{\frac{\text { Internal flux } \text { Intest }_{\text {Internal flux }}}{\text { motion }}}=1.000000000006
\end{aligned}
$$

The Internal flux motion of the clock in motion is lower meaning the clock ticks slower as expected. Using the Lorentz factor, the results are the same.

$$
\begin{aligned}
& \text { Lorentz factor denominator }=\sqrt{1-\frac{(1000 \mathrm{~m} / \mathrm{s})^{2}}{c^{2}}}=0.999999999994 \\
& \text { Lorent } z \text { factor }=\frac{1}{\sqrt{1-\frac{(1000 \mathrm{~m} / \mathrm{s})^{2}}{c^{2}}}}=1.000000000006
\end{aligned}
$$

The result of the Internal flux ratio in equation (B.2) can be used in equation (1). As expected, the number of cycles decreases when the clock is in motion.

$$
0.999999999994 * 9192631770 \text { cycles }=9192631769.9489 \text { cycles }
$$

Fig. B.3. represents the correlation between the Internal flux ratio and the Lorentz factor denominator. Fig. B.4. represents the correlation between the Internal flux ratio inverted and the Lorentz factor. There is, in both figures, an exact match between the curves. Fig. B.5. shows that a proportional change in masses in the compared systems does not affect the results. However, if the two compared systems have different masses, 
the results will be affected. The Lorentz factor does not include the mass because only the speed is sufficient to calculate a time dilation.

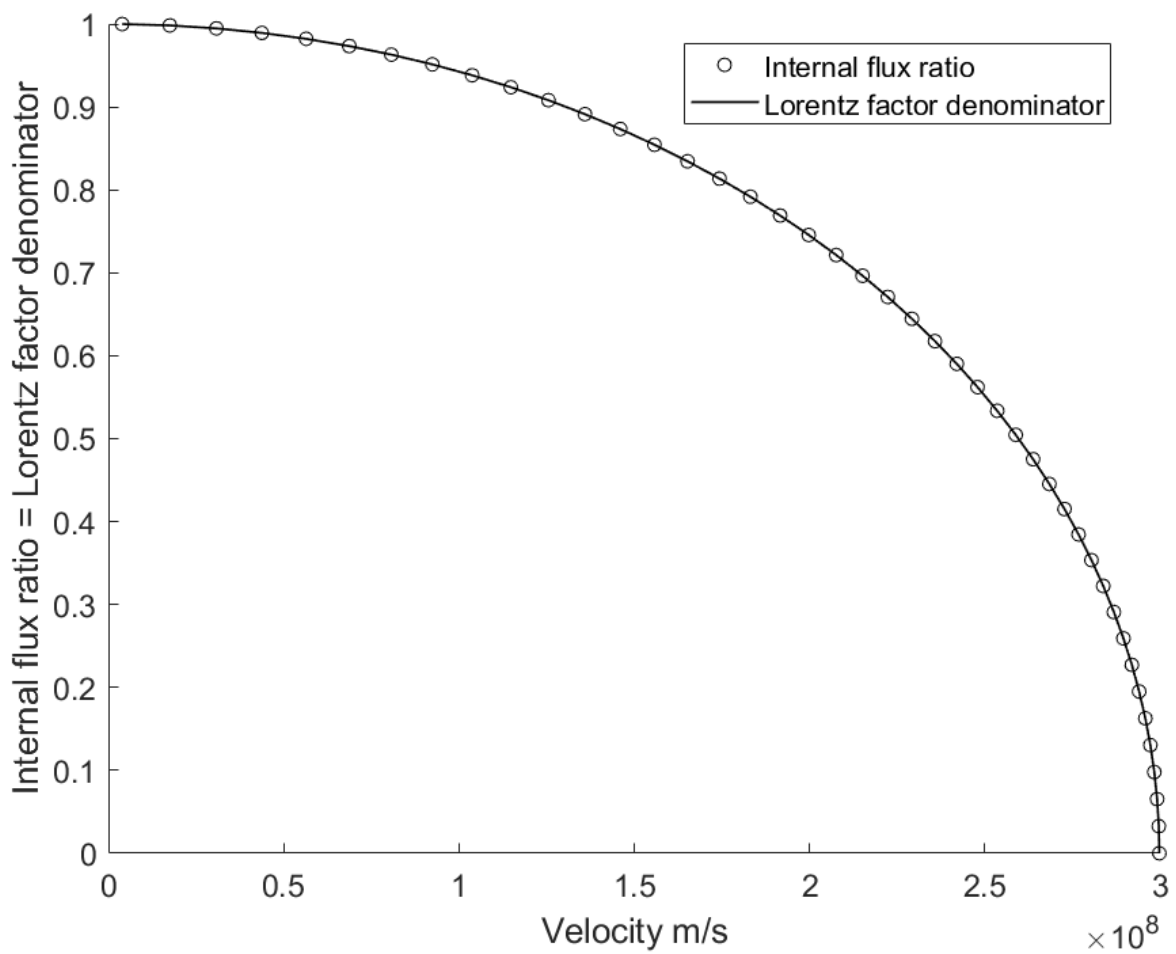

Fig. B.3. Correlation between the Internal flux ratio and the Lorentz factor denominator

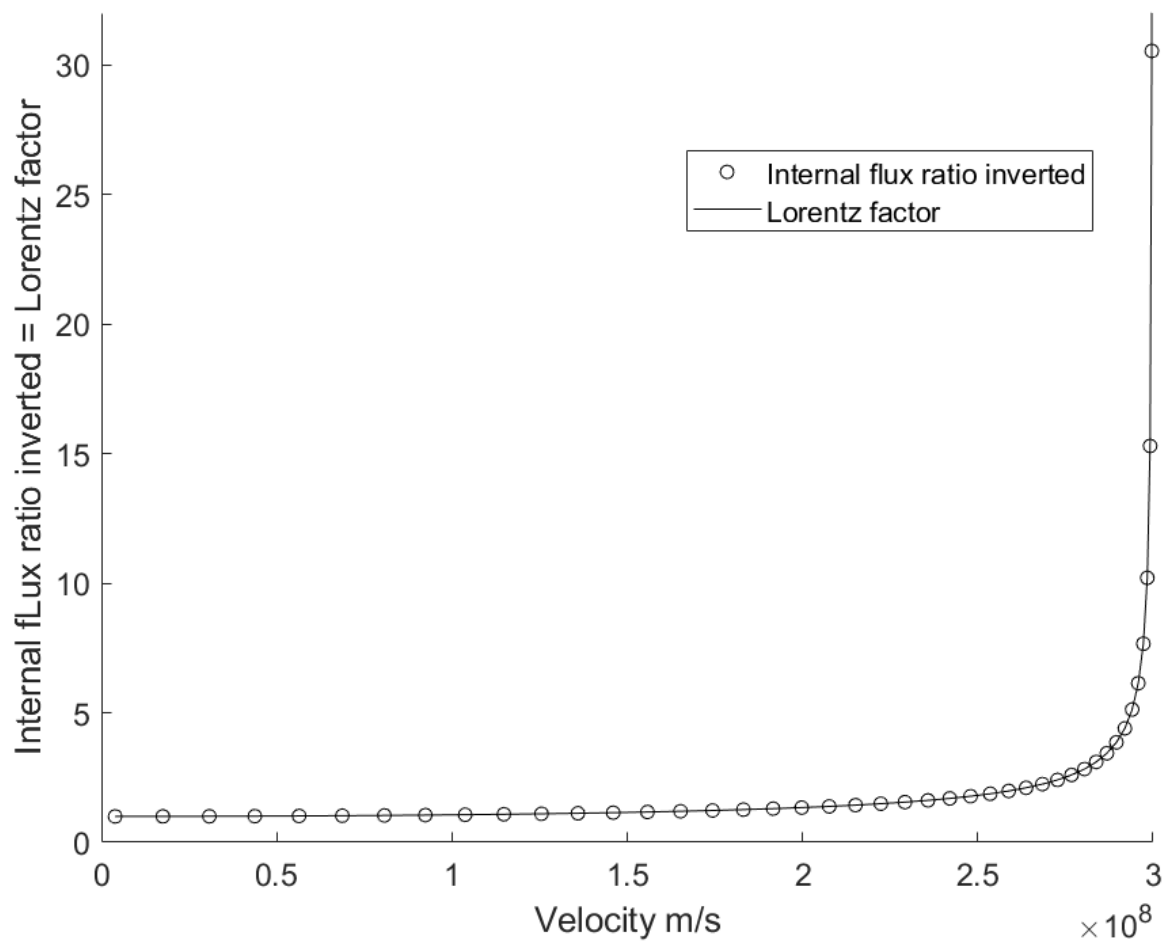

Fig. B.4. Correlation between the Internal flux ratio inverted and the Lorentz factor 


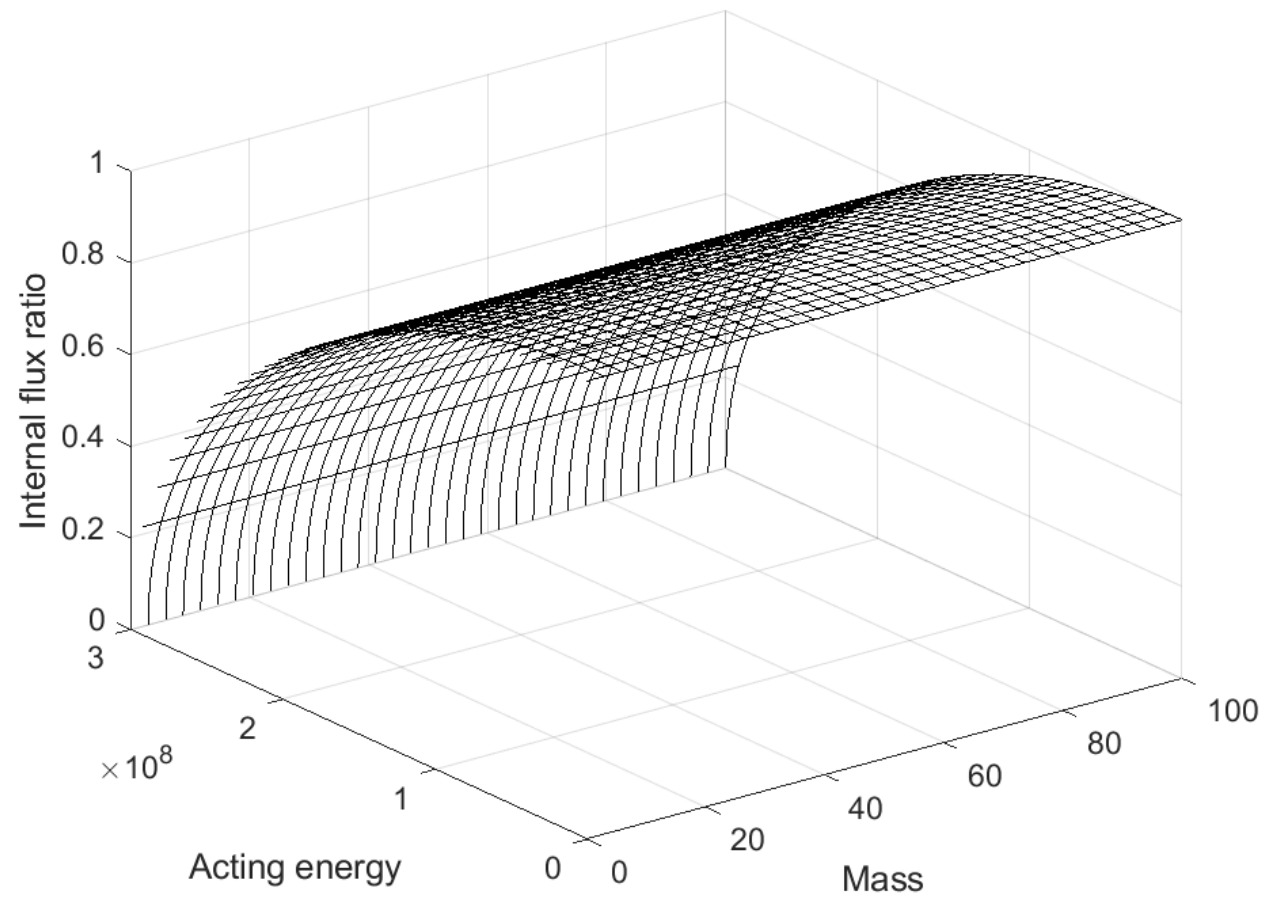

Fig. B.5. Invariance when the masses change proportionally in both systems

\section{Appendix B.2. Gravitational time dilation correlation}

We consider three energy systems, the earth as the reference point, one clock in motion, and a clock at rest.
Mass clock $_{1}$
$1 \mathrm{~kg}$
Mass clock $_{2}$
$1 \mathrm{~kg}$
Mass earth
$5.972 E 24 \mathrm{~kg}$
Altitude clock 1
$6940000 m$
Altitude clock2
$6740000 m$
Attractive energy $y_{\text {earth }} \rightarrow$ clock 1
$5.74317 \mathrm{~J}$
Attractive energy $y_{\text {earth }} \rightarrow$ clock 2
$5.91359 \mathrm{~J}$
Gravitational constant $G$

$$
6.67408 e-11 \mathrm{~m}^{3} \mathrm{~kg}^{-1} \mathrm{~s}^{-2}
$$

The attractive energy produced between two masses is given by the gravitational potential energy formula.

$$
\text { Attractive energy (at distance } r)=G \frac{M_{1} M_{2}}{r}
$$

However, the Acting energy that makes the equation works is the double of the Attractive energy.

$$
\text { Acting energy }=2 * G \frac{M_{1} M_{2}}{r}
$$


We subtract the Acting energy for each clock to find their respective Internal flux and we calculate the Internal flux ratio between the two clocks.

$$
\begin{aligned}
& \text { Internal flux } \text { clock } 1=\sqrt{\frac{M_{\text {clock } 1^{2}-(2 * 5.7432 E 7 ~ J)}}{M_{\text {clock } 1}}}=299792457.8084 \\
& \text { Internal flux } \text { clock } 2=\sqrt{\frac{M_{\text {clock } 2^{c^{2}}-(2 * 5.9136 E 7 ~ J)}}{M_{\text {clock } 2}}}=299792457.8027
\end{aligned}
$$

We compare the two internal fluxes.

$$
\text { Internal flux ratio }=\sqrt{\frac{\text { Internal flux } \text { clock } 1}{\text { Internal flux } x_{\text {clock } 2}}}=1.000000000019
$$

The Internal flux ratio is greater than 1 . When 1 second has elapsed on clock2, then 1.000000000019 seconds will have elapsed on clock1, meaning the time of clock1 is faster. This result is consistent with the gravitational time dilation expected in higher altitude. We use as a comparison the official gravitational time dilation equation.

$$
\begin{aligned}
& t_{0}=t_{f} * \sqrt{1-\frac{2 G M}{r c^{2}}} \\
& t_{\text {clock } 1}=1 * \sqrt{1-\frac{2 G * 5.972 E 24}{6940000 * c^{2}}}=0.999999999361 \\
& t_{\text {clock } 2}=1 * \sqrt{1-\frac{2 G * 5.972 E 24}{6740000 * c^{2}}}=0.999999999342 \\
& \frac{t_{\text {clock } 1}}{t_{\text {clock } 2}}=1.000000000019
\end{aligned}
$$

The result is the same and has the same meaning as equation B.8. Using the Internal flux ratio equation (21), the gravitational time dilation equation can be derived. In the following derivation, $M_{1}$ is any mass at a distance $r$ from earth and $M_{E}$ is the mass of earth.

$$
\begin{aligned}
\frac{\text { Internal flux } 1}{\text { Internal flux } 2} & =\frac{\sqrt{M_{1} c^{2}-2 G \frac{M_{1} M_{E}}{r}}}{\sqrt{M_{1} c^{2}}}=\sqrt{\frac{M_{1} c^{2}-2 G \frac{M_{1} M_{E}}{r}}{M_{1} c^{2}}} \\
& =\sqrt{\frac{M_{1} c^{2}}{M_{1} c^{2}}-\frac{2 G M_{1} M_{E}}{r M_{1} c^{2}}}=\sqrt{1-\frac{M_{1}}{M_{1}} \frac{2 G M_{E}}{r c^{2}}}=\sqrt{1-\frac{2 G M_{E}}{r c^{2}}}
\end{aligned}
$$

Fig. B.6. represents the correlation between the internal flux ratio and the gravitational time dilation equation. There is an exact match between the curves. 


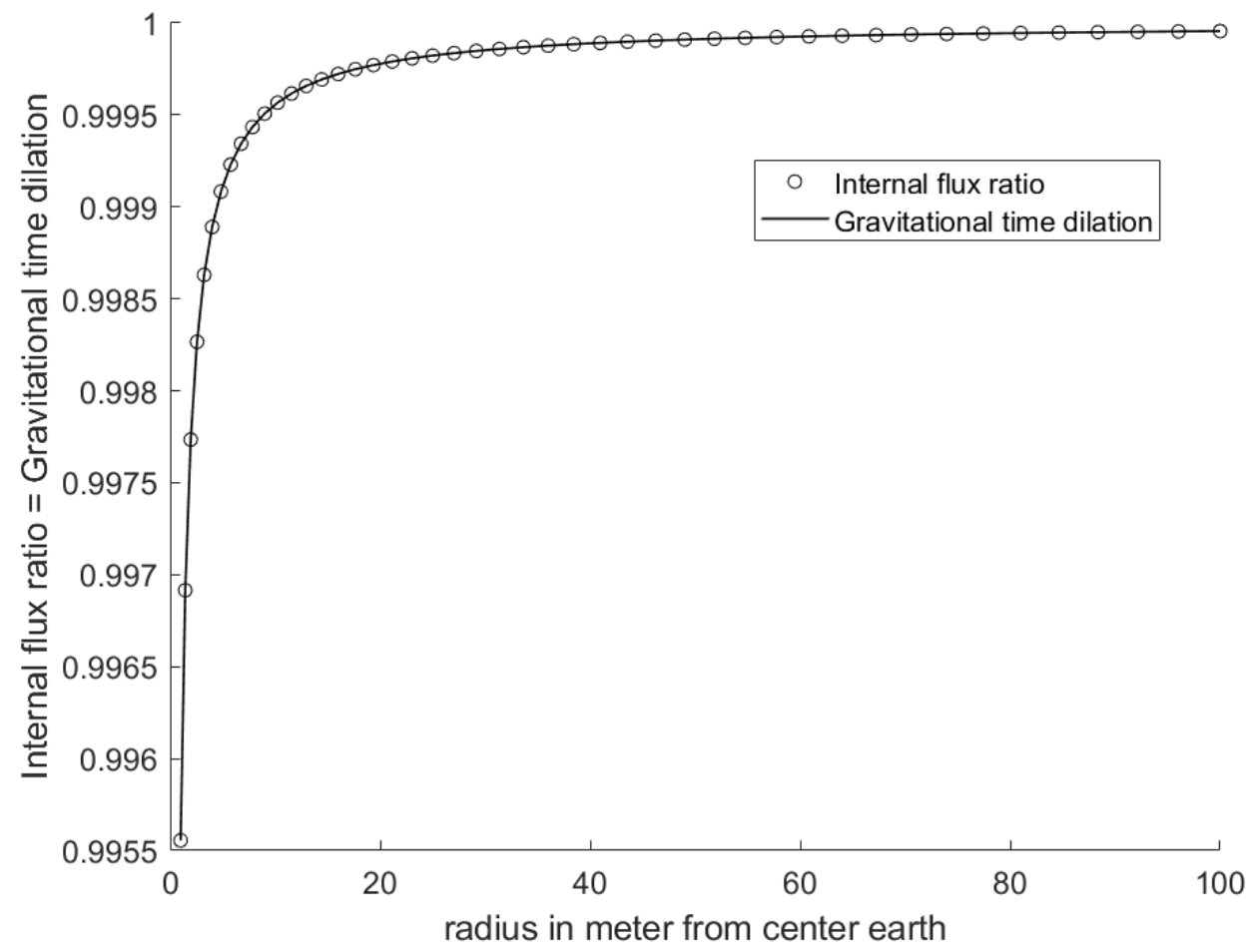

Fig. B.6. Correlation between the internal flux ratio and the gravitational time dilation equation

\section{Appendix C. Supplementary data}

The scripts to calculate the results sections 4.2 to 4.4 and the results in Appendix B.1 and Appendix B.2 are available in the OSF repository at https://osf.io/m6u57 


\section{References}

Arstila, V., 2012. Time slows down during accidents. Frontiers in Psychology doi:10.3389/ fpsyg. 2012.00196.

Buhusi, C.V., Meck, W.H., 2009. Relativity theory and time perception: Single or multiple clocks? PLoS ONE doi:10.1371/journal.pone.0006268.

Capek, M., 1987. The Conflict between the Absolutist and the Relational Theory of Time before Newton. Journal of the History of Ideas doi:10.2307/2709689.

Church, R.M., 1984. Properties of the Internal Clock. Annals of the New York Academy of Sciences 423, 566-582. doi:10.1111/j.1749-6632.1984.tb23459.x.

Davydenko, M., Peetz, J., 2017. Time grows on trees: The effect of nature settings on time perception. Journal of Environmental Psychology doi:10.1016/j.jenvp.2017.09.003.

Droit-Volet, S., 2013. Time perception, emotions and mood disorders. doi:10.1016/j . jphysparis.2013.03.005.

Droit-Volet, S., Chaulet, M., Dambrun, M., 2018. Time and Meditation: When Does the Perception of Time Change with Mindfulness Exercise? Mindfulness doi:10.1007/ s12671-018-0903-6.

Droit-Volet, S., Fanget, M., Dambrun, M., 2015. Mindfulness meditation and relaxation training increases time sensitivity. Consciousness and Cognition doi:10.1016/j . concog. 2014.10 .007$.

Edwards, A.M., McCormick, A., 2017. Time perception, pacing and exercise intensity: maximal exercise distorts the perception of time. Physiology and Behavior doi:10.1016/ j.physbeh.2017.08.009.

Einstein, A., 1905a. Does the inertia of a body depend upon its energy-content. Annalen der Physik .

Einstein, A., 1905b. On the electrodynamics of moving bodies (Zur Elektrodynamik bewegter Körper). Annalen der Physik .

Einstein, A., 1916. The Foundation of the General Theory of Relativity (Die Grundlage der allgemeinen Relativitätstheorie). Annalen der Physik doi:10.1002/andp.19163540702.

Einstein, A., 1920. Relativity: The Special \& General Theory.

Fayolle, S., Gil, S., Droit-Volet, S., 2015. Fear and time: Fear speeds up the internal clock. Behavioural Processes doi:10.1016/j . beproc.2015.09.014. 
Ghaderi, A.H., 2019. Heat transfer, entropy and time perception: Toward finding a possible relation between subjective and objective time. Medical Hypotheses doi:10.1016/j. mehy. 2018.11.018.

Gibbon, J., CHURCH, R.M., MECK, W.H., 1984. Scalar Timing in Memory. Annals of the New York Academy of Sciences doi:10.1111/j.1749-6632.1984.tb23417.x.

Hass, J., Durstewitz, D., 2016. Time at the center, or time at the side? Assessing current models of time perception. doi:10.1016/j.cobeha.2016.02.030.

Kent, L., van Doorn, G., Hohwy, J., Klein, B., 2019. Bayes, time perception, and relativity: The central role of hopelessness. Consciousness and Cognition doi:10.1016/j.concog. 2019.01 .012$.

Magistretti, P.J., Allaman, I., 2015. A Cellular Perspective on Brain Energy Metabolism and Functional Imaging. doi:10.1016/j.neuron.2015.03.035.

Mioni, G., Grondin, S., Bardi, L., Stablum, F., 2020. Understanding time perception through non-invasive brain stimulation techniques: A review of studies. Behavioural Brain Research doi:10.1016/j.bbr.2019.112232.

Shuler Jr., R.L., 2014. The Twins Clock Paradox History and Perspectives. Journal of Modern Physics doi:10.4236/jmp.2014.512108.

Tanenbaum, A.S., Bos, H., 2014. Modern Operating Systems. doi:10.1142/ S0129183108012261, arXiv:0710.2023.

Taylor, B.N., Thompson, A., Cd Mol Kg, A.K., Si, S., 2008. The International System of Units (SI) NIST Special Publication 3302008 Edition. Technical Report. doi:10.1119/ 1.2351766 .

Thomas, E., 2015. Henry More and the development of absolute time. Studies in History and Philosophy of Science Part A doi:10.1016/j.shpsa.2015.06.003.

Thönes, S., Stocker, K., 2019. A standard conceptual framework for the study of subjective time. doi:10.1016/j. concog. 2019.04.004.

Treisman, M., 1963. Temporal discrimination and the indifference interval. Implications for a model of the "internal clock". Psychological monographs doi:10.1037/h0093864.

Xu, S., David, P., 2018. Distortions in time perceptions during task switching. Computers in Human Behavior doi:10.1016/j.chb.2017.11.032. 